\title{
String theory integrands and supergravity divergences
}

\author{
Boris Pioline \\ Laboratoire de Physique Théorique et Hautes Énergies (LPTHE), \\ CNRS and Sorbonne Université, UMR 7589, \\ 4 place Jussieu, F-75005 Paris, France \\ E-mail: pioline@lpthe.jussieu.fr
}

ABSTRACT: At low energies, interactions of massless particles in type II strings compactified on a torus $T^{d}$ are described by an effective Wilsonian action $\mathcal{S}(\Lambda)$, consisting of the usual supergravity Lagrangian supplemented by an infinite series of higher-derivative vertices, including the much studied $\nabla^{4 p+6 q} \mathcal{R}^{4}$ gravitational interactions. Using recent results on the asymptotics of the integrands governing four-graviton scattering at genus one and two, I determine the $\Lambda$-dependence of the coefficient of the above interaction, and show that the logarithmic terms appearing in the limit $\Lambda \rightarrow 0$ are related to UV divergences in supergravity amplitudes, augmented by stringy interactions. This provides a strong consistency check on the expansion of the integrand near the boundaries of moduli space, in particular it elucidates the appearance of odd zeta values in these expansions. I briefly discuss how these logarithms are reflected in non-analytic terms in the low energy expansion of the string scattering amplitude.

KEYwords: String Duality, Scattering Amplitudes, Extended Supersymmetry

ARXIV EPRINT: 1810.11343 


\section{Contents}

1 Introduction 1

2 Scale dependence of truncated modular integrals 4

2.1 Genus-one 4

2.2 Genus-two 7

2.3 Genus three 13

3 Matching with supergravity divergences $\quad 14$

$\begin{array}{ll}3.1 & \text { Qualitative analysis of UV divergences in supergravity } \\ \end{array}$

$\begin{array}{lll}3.2 & \text { Combining genus-one and genus-two scale-dependent terms } & 19\end{array}$

4 Discussion $\quad 23$

A Constraints on $\nabla^{10} \mathcal{R}^{4}$ interactions $\quad 26$

B Some properties of modular local polynomials 28

\section{Introduction}

String theory is a manifestly UV finite theory of perturbative quantum gravity. Indeed, the $h$-th term in the weak coupling expansion of a closed string amplitude with $n$ external legs involves an integral over the moduli space $\mathcal{M}_{h, n}$ of Riemann surfaces of genus $h$ with $n$ punctures [1] — or rather, the moduli space $\mathfrak{M}_{h, n}$ of super-Riemann surfaces, which projects (albeit non-holomorphically) onto $\mathcal{M}_{h, n}[2,3]$. The latter is non-compact but its only boundaries correspond to low energy processes where the string propagates for a very long proper time. As a result, the analytic properties of scattering amplitudes of massless excitations around string vacua with extended supersymmetry are identical to those computed in supergravity, supplemented with an infinite series of higher-derivative interactions induced by the exchange of massive string modes [4]. In particular, string amplitudes around vacua of the form $\mathbb{R}^{1, D-1} \times X_{d}$, where $X_{d}$ is a compact manifold of dimension $d=10-D$, are IR finite when $D>4$, and have the standard infrared divergences of supergravity in $D \leq 4$. In either case, they are non-analytic functions of the Mandelstam variables $s_{i j}=-2 k_{i} \cdot k_{j}$ in the low energy limit $s_{i j} \rightarrow 0$, due to the exchange of massless supergravity modes.

In order to separate these infrared singularities from genuinely stringy effects, it is convenient to decompose the moduli space $\mathcal{M}_{h, n}$ into a disjoint union $\mathcal{M}_{h, n}(\Lambda) \sqcup \mathcal{N}_{h, n}(\Lambda)$, where $\mathcal{M}_{h, n}(\Lambda)$ is the subset of $\mathcal{M}_{h, n}$ where the proper time $t$ associated to any handle is less than $L=1 /\left(\alpha^{\prime} \Lambda^{2}\right)$, and $\mathcal{N}_{h, n}(\Lambda)$ is its complement. Here, $\Lambda$ is an arbitrary intermediate 
scale, much larger than the energy of the external particles but much smaller than the string scale $1 / \sqrt{\alpha^{\prime}}$. The integral over the compact component $\mathcal{M}_{h, n}(\Lambda)$ is now analytic as $s_{i j} \rightarrow 0$, and the resulting contribution to the amplitude (which we refer to as the truncated string amplitude) can be described by local interactions in an effective Wilsonian action $\mathcal{S}(\Lambda)$ for the supergravity modes (see [5] for an alternative definition of the Wilsonian action in string field theory), which reduces to the 1-particle irreducible (1PI) effective action as $\Lambda \rightarrow 0$. The integral over the non-compact complement $\mathcal{N}_{h, n}(\Lambda)$ can be interpreted as a sum of quantum field theory amplitudes with $\ell<h$ loops computed from the action $\mathcal{S}(\Lambda)$, with a UV cut-off $t>L$ on the proper time associated to each loop (and possibly an IR cut-off $t<1 /\left(\alpha^{\prime} \mu^{2}\right)$ when $\left.D \leq 4\right)$. The sum of the two contributions is by construction independent of $L$, with the variation of $L$ defining a renormalization group flow on the coefficients of the local couplings in the action $\mathcal{S}(\Lambda)$. Thus, the dependence of the truncated string amplitude on the sliding scale $\Lambda$ (which acts as an IR cut-off in string theory) contains information about the dependence of the field theory amplitudes on the UV cut-off. The former is in turn governed by the behavior of the integrand of the string amplitude near the boundaries of $\mathcal{M}_{h, n}$.

The goal of this note will be to demonstrate this connection explicitly in the case of the four-graviton scattering amplitudes in type II strings compactified on a torus $T^{d}$, extending the earlier analysis of [6] to higher order in the derivative expansion. In the limit where the Mandelstam variables $s=s_{12}, t=s_{13}, u=s_{14}$ go to zero, this amplitude can be expanded as $[7]$

$$
\mathcal{A}(s, t, u)=\mathcal{A}_{\text {non.an. }}(s, t, u ; L)+\sum_{p, q=0}^{\infty} \mathcal{E}_{(p, q)}^{(d)}(\varphi, L) \frac{\sigma_{2}^{p} \sigma_{3}^{q}}{p ! q !} \mathcal{R}^{4} \ell_{D}^{8}
$$

where $\sigma_{n}=\left(s^{n}+t^{n}+u^{n}\right) \ell_{D}^{2 n} / 4^{n}$ with $n=2,3$ form a basis of symmetric functions of $s, t, u$ subject to the momentum conservation condition $\sigma_{1}=0$. Here, $\ell_{D}$ is the $D$-dimensional Planck length, $\mathcal{R}^{4}$ is a polynomial of degree 8 in momenta and degree 4 in the polarization tensor of the gravitons, and $\mathcal{E}_{(p, q)}(\varphi, L)$ are the coefficients of local interactions of the form $\ell_{D}^{8-D-2 p-3 q} \nabla^{4 p+6 q} \mathcal{R}^{4}$ in the Wilsonian action $\mathcal{S}(\Lambda)$ in Einstein frame. These coefficients are functions of the moduli fields $\varphi$ parametrizing the constant metric and gauge forms on the internal torus as well as the $D$-dimensional string coupling $g_{D}$. In maximally supersymmetric vacua, $\varphi$ is valued in the symmetric space $\mathcal{M}_{D}=E_{d+1}(\mathbb{Z}) \backslash E_{d+1} / K_{d+1}$, where $E_{d+1}$ is the split reductive Lie group of Cartan type $E_{d+1}, K_{d+1}$ its maximal compact subgroup and $E_{d+1}(\mathbb{Z})$ an arithmetic subgroup of $E_{d+1}$ known as the U-duality group [8].

The first term $\mathcal{A}_{\text {non.an. }}(s, t, u ; L)$ in (1.1) corresponds to the integral over the domains $\mathcal{N}_{h, n}(\Lambda)$, and can be viewed as a supergravity amplitude with UV cut-off $\Lambda=1 / \sqrt{\alpha^{\prime} L}$, involving the usual massless propagators and supergravity vertices, supplemented with higher derivative interactions. In the limit $L \rightarrow \infty$, the coefficients $\mathcal{E}_{(p, q)}^{(d)}(\varphi, L)$ are typically divergent, but these divergences are cancelled by $L$-dependent contributions from $\mathcal{A}_{\text {non.an. }}(s, t, u ; L)$ proportional to $\sigma_{2}^{p} \sigma_{3}^{q} \mathcal{R}^{4}$. After subtracting these divergent terms, one obtains finite, 'renormalized' automorphic functions $\mathcal{E}_{(p, q)}^{(d)}(\varphi)$ on $\mathcal{M}_{D}$, which have been intensively studied in recent years (see e.g. [9] for a recent entry point in this vast literature). We stress that the adjective 'renormalized' is not related to the usual renormalization pre- 
scription in quantum field theory, but instead refers to a specific prescription for separating the analytic and non-analytic part of the 1PI effective action.

As indicated earlier, the coefficient $\mathcal{E}_{(p, q)}^{(d)}(\varphi, L)$ of the $\nabla^{4 p+6 q} \mathcal{R}^{4}$ effective interaction is given, up to a priori unknown non-perturbative effects, by a genus expansion

$$
\mathcal{E}_{(p, q)}^{(d)}(\varphi, L)=g_{D}^{\frac{2 D-8 p-12 q-16}{D-2}} \sum_{h=0}^{\infty} g_{D}^{2 h-2} \mathcal{E}_{(p, q)}^{(d, h)}(\varphi, L)+\mathcal{O}\left(e^{-2 \pi / g_{D}}\right),
$$

where $\mathcal{E}_{(p, q)}^{(d, h)}(\varphi, L)$ is given by an integral over the truncated moduli space $\mathcal{M}_{h, 4}(\Lambda)$, and the prefactor arises when translating from string frame to Einstein frame (or equivalently, when expressing the string length $\ell_{s}$ in terms of the Planck length $\left.\ell_{D}=\ell_{s} g_{D}^{2 /(D-2)}\right)$. The renormalized coupling $\mathcal{E}_{(p, q)}^{(d)}(\varphi)$ has a similar genus expansion, except for an additional contribution $\mathcal{E}_{(p, q)}^{(d), \text { non.an. }}$ which is non-analytic at $g_{D}=0$ and arises in the process of translating $\mathcal{A}_{\text {non.an. }}(s, t, u ; L)$ into Einstein frame $[6,9,10]$. At tree-level, the coefficients are pure constants known to arbitrary order, the first few ones being $[7,11]$

$$
\mathcal{E}_{(0,0)}^{(d, 0)}=2 \zeta(3), \quad \mathcal{E}_{(1,0)}^{(d, 0)}=\zeta(5), \quad \mathcal{E}_{(0,1)}^{(d, 0)}=\frac{2}{3} \zeta(3)^{2}, \quad \mathcal{E}_{(2,0)}^{(d, 0)}=\zeta(7), \quad \mathcal{E}_{(1,1)}^{(d, 0)}=\frac{2}{3} \zeta(3) \zeta(5) .
$$

At genus-one, the scattering amplitude is known since [12] and its low energy expansion has been studied to high order in the $\alpha^{\prime}$ expansion $[7,13,14]$. After integrating over the location of the four vertex operators, the coefficients $\mathcal{E}_{(p, q)}^{(d, 1)}(\varphi, L)$ are given by integrals of modular graph functions [15] over the truncated fundamental domain $\mathcal{F}_{1}(L)$ in the Poincaré upper-half plane $\mathcal{H}_{1}$. The cancellation of the $L$-dependent terms against the non-analytic part $\mathcal{A}_{\text {non.an. }}(s, t, u ; L)$ was studied in detail in [13], primarily in dimensions $D=10$ and $D=9$, using unitarity to determine the threshold contributions.

The genus-two amplitude was computed in a heroic effort [16-18] and its low energy expansion was investigated in [19-21]. The coefficients $\mathcal{E}_{(p, q)}^{(d, 2)}(\varphi, L)$ are again given by integrals of genus-two modular graph functions [21] over the truncated fundamental domain $\mathcal{F}_{2}(L)$ in the Siegel upper-half plane [22]. Recently, an extensive analysis of the asymptotic behavior of the genus-two integrands near the separating and non-separating degeneration limits was carried out up to order $\nabla^{8} \mathcal{R}^{4}$ [21, 23], extending earlier work on the supergravity limit of genus-two string amplitudes [24].

In section 2 , we shall use these asymptotics to compute the $L$-dependence of the truncated amplitude at genus-two, and extract the coefficients of the logarithmic terms in various dimensions. In section 3 we compare these results to the structure of ultraviolet divergences in maximally supersymmetric supergravity, obtained up to three loop in $[25$, 26]. We shall find quantitative agreement, providing a strong check on the asymptotics computed in [23], as well as elucidating the physical origin of the transcendental coefficients which appear in these expansions. In section 4, we briefly discuss the implications of these logarithms for the non-analytic part of the amplitude. The detailed study of the cancellation of the $L$-dependent terms against the non-analytic part of the 1PI effective action is left for future work. While we restrict attention to interactions up to order $\nabla^{8} \mathcal{R}^{4}$ in the body of the paper, the constraints on the asymptotics of the genus-two integrand at 
order $\nabla^{10} \mathcal{R}^{4}$ are investigated in appendix A. Finally, some details about a class of modular functions appearing in the tropical limit of genus two string amplitudes are collected in appendix B.

Note added in v2. In the first release of this work, the truncated moduli space $\mathcal{M}_{h, n}(\Lambda)$ was defined as the subspace of $\mathcal{M}_{h, n}$ where the length of any closed geodesic measured with respect to the hyperbolic metric is less than $L=1 /\left(\alpha^{\prime} \Lambda^{2}\right)$. This definition does not make sense since closed geodesics typically have unbounded length. A more sensible definition is to put a lower cut-off $\ell>\epsilon$ on the length of the shortest closed geodesic (also known as the systole). Indeed, from the point of view of hyperbolic metric, all boundaries of $\mathcal{M}_{h, n}$ are associated to the vanishing $\ell \rightarrow 0$ of the length of a closed geodesic (homologically trivial in the separating case, or non-trivial in the non-separating case). In the vicinity of a locus where $\ell \rightarrow 0$, the geometry is well approximated by the wedge $\left\{\frac{\ell}{2}<\arg z<\pi-\frac{\ell}{2}\right\}$ inside the Poincaré upper half-plane, modded out by dilations $z \mapsto e^{\ell} z-$ a model known as the standard collar (see e.g. [46, 47]). This region is conformal to the cone $x y=\tau$ with $\log |\tau| \sim-2 \pi^{2} / \ell$. As the length $\ell$ of the geodesic along the imaginary axis shrinks to 0 size, the length $\ell^{\prime}=\int_{\ell / 2}^{\pi-\ell / 2} \frac{\mathrm{d} \theta}{\sin \theta}$ of a geodesic at any fixed radial distance diverges as $\ell^{\prime} \sim-2 \log \ell$. A lower cut-off $\ell>\epsilon$ therefore implies an upper bound $\ell^{\prime}<-2 \log \epsilon$ on the length of geodesics going through the node. In view of the relation $\Omega_{i j} \sim \frac{1}{2 \pi \mathrm{i}} \log \tau$ between the gluing parameter $\tau$ and the relevant entry $\Omega_{i j}$ in the period matrix (in the case of a non-separating degeneration) [48], one readily sees that a lower cut-off $\ell>\epsilon$ implies an upper bound $t \sim \operatorname{Im} \Omega_{i j}<\pi / \epsilon$ on the proper time associated to the long handle (for a separating degeneration, the relevant entry $\Omega_{i j}$ is proportional to $\tau$, but exponential in proper time, leading to the same conclusion). Thus, we can define $\mathcal{M}_{h, n}(\Lambda)$ as the subspace of $\mathcal{M}_{h, n}$ where closed geodesics with respect to the hyperbolic metric have length greater than $\pi / L=\pi \alpha^{\prime} \Lambda^{2}$. It would be interesting to give a similar characterization in terms of the minimal area metric which is more commonly used in the string field theory literature [49].

\section{Scale dependence of truncated modular integrals}

In this section, we discuss the scale dependence of the modular integrals computing the coefficient $\mathcal{E}_{(p, q)}^{(d, h)}(\varphi, L)$ of the four-graviton effective interaction in type II string theory compactified on a torus $T^{d}$, at genus $h=1$ and $h=2$. We also briefly review the genus three result at leading order in the derivative expansion.

\subsection{Genus-one}

By expanding the integrand of the genus-one four-graviton scattering in powers of the Mandelstam variables at fixed value of the complex modulus $\tau$ of the worldsheet torus, and integrating over the position of the four vertex operators, one finds a contribution to the effective coupling $\mathcal{E}_{(p, q)}^{(d)}$ of the form [13]

$$
\mathcal{E}_{(p, q)}^{(d, 1)}(\varphi, L)=\pi \int_{\mathcal{F}_{1}(L)} \mathrm{d} \mu_{1} \Gamma_{d, d, 1}(\tau ; \varphi) \mathcal{B}_{(p, q)}^{(1)}(\tau),
$$


where $\mathrm{d} \mu_{1}=2 \mathrm{~d} \tau_{1} \mathrm{~d} \tau_{2} / \tau_{2}^{2}$ is the invariant measure on the Poincaré upper-half plane, $\Gamma_{d, d, 1}$ is the genus-one Siegel-Narain theta series

$$
\Gamma_{d, d, 1}(\tau ; \varphi)=\tau_{2}^{d / 2} \sum_{\left(m_{i}, n^{i}\right) \in \mathbb{Z}^{2 d}} e^{-\pi \tau_{2}\left[\left(m_{i}+B_{i j n j} G^{i k}\left(m_{k}+B_{k l} n^{l}\right)+n^{i} G_{i j} n^{j}\right]+2 \pi \mathrm{i} m_{i} n^{i} \tau_{1}\right.},
$$

where $G_{i j}$ is the constant metric on the torus $T^{d}, G^{i j}$ its inverse and $B_{i j}$ the Kalb-Ramond two-form - all being functions of the moduli $\varphi$. The factor $\mathcal{B}_{(p, q)}^{(1)}$ in the integrand is a nonholomorphic modular function of $\tau$, given for low values of $(p, q)$ by [13] [14, (7.17), (7.28)]: ${ }^{1}$

$$
\mathcal{B}_{(0,0)}^{(1)}=1, \quad \mathcal{B}_{(1,0)}^{(1)}=E_{2}, \quad \mathcal{B}_{(0,1)}^{(1)}=\frac{1}{3}\left(5 E_{3}+\zeta(3)\right), \quad \mathcal{B}_{(2,0)}^{(1)}=\frac{1}{12}\left(D_{4}+9 E_{2}^{2}+6 E_{4}\right),
$$

where $E_{a}(\tau)$ are the usual non-holomorphic Eisenstein series, normalized such that $E_{a}=$ $2 \tau_{2}^{a} \zeta(2 a) / \pi^{a}+\mathcal{O}\left(\tau_{2}^{1-a}\right)$, while $D_{4}(\tau)$ is the modular graph function defined in [14, section 5.3].

Finally, $\mathcal{F}_{1}(L)$ is a truncated version of the fundamental domain for the action of $\mathrm{SL}(2, \mathbb{Z})$ on the upper half plane parametrized by $\tau=\tau_{1}+\mathrm{i} \tau_{2} \in \mathcal{H}_{1}$, introduced in the present context in [7],

$$
\mathcal{F}_{1}(L)=\left\{-\frac{1}{2}<\tau_{1}<\frac{1}{2}, \quad|\tau|>1, \quad \tau_{2}<L\right\},
$$

where $L$ is a large but finite constant. The truncation $\tau_{2}<L$ ensures that the proper time around the loop is less than $L$ (in units where the proper length of the string is normalized to 1 ). We shall refer to integrals of modular forms over the truncated fundamental domain as 'truncated modular integrals'. Clearly, one could choose a different fundamental domain for the action of $\mathrm{SL}(2, \mathbb{Z})$, but then the truncation should be appropriately modified, such that it covers the same domain as $\mathcal{F}_{1}(L)$ on the quotient $\mathcal{H}_{1} / \mathrm{SL}(2, \mathbb{Z})$.

Our purpose will be to extract the dependence of the truncated modular integral (2.1) on the scale $L$ as it is taken to infinity. The simplest way is to compute the derivative with respect to $L$,

$$
L \partial_{L} \mathcal{E}_{(p, q)}^{(d, 1)}(\varphi, L)=\frac{2 \pi}{L} \int_{-1 / 2}^{1 / 2} \mathrm{~d} \tau_{1}\left[\Gamma_{d, d, 1}(\tau ; \varphi) \mathcal{B}_{(p, q)}^{(1)}(\tau)\right]_{\tau_{2}=L}
$$

In the limit $\tau_{2}=L \rightarrow \infty$, we can approximate $\Gamma_{d, d, 1}(\tau ; \varphi) \sim \tau_{2}^{d / 2}$ with exponential accuracy. We shall assume that in this limit, the constant term of the integrand $\mathcal{B}_{(p, q)}^{(1)}$ has a finite Laurent series expansion

$$
\int_{-1 / 2}^{1 / 2} \mathrm{~d} \tau_{1} \mathcal{B}_{(p, q)}^{(1)}(\tau)=\sum_{i=1}^{\ell} c_{i} \tau_{2}^{\sigma_{i}}+\mathcal{O}\left(e^{-2 \pi \tau_{2}}\right),
$$

up to exponentially suppressed corrections. Indeed, in the cases of interest, $\mathcal{B}_{(p, q)}^{(1)}$ is a modular graph function of weight ${ }^{2} w=2 p+3 q$ with a Laurent expansion of the form $(2.6)$

\footnotetext{
${ }^{1}$ The factor of $p ! q !$ is not included in [14]; to compare with [9], note that $E_{2}^{*}=\frac{1}{2} E_{2}, E_{3}^{*}=E_{3}$.

${ }^{2}$ Here $w$ denotes the transcendental weight, while the modular weight vanishes.
} 
with $\sigma_{i} \in[1-w, \ldots, w]$. Under this assumption, we find

$$
L \partial_{L} \mathcal{E}_{(p, q)}^{(d, 1)}(\varphi, L)=2 \pi \sum_{i=1}^{\ell} c_{i} L^{\frac{d}{2}+\sigma_{i}-1} .
$$

Upon integrating, it follows that the truncated modular integral (2.1) behaves as $L \rightarrow \infty$ as

$$
e_{(p, q)}^{(d, 1)}(L)=2 \pi\left(\sum_{\sigma_{i}>1-\frac{d}{2}} c_{i} \frac{L^{\frac{d}{2}+\sigma_{i}-1}}{\frac{d}{2}+\sigma_{i}-1}+\sum_{\sigma_{i}=1-\frac{d}{2}} c_{i} \log L\right)
$$

up to an additive constant. Following the terminology of [27], we define the 'renormalized' integral $\mathcal{E}_{(p, q)}^{(d, 1)}$ by subtracting the power-like terms before taking the limit $L \rightarrow \infty$,

$$
\mathcal{E}_{(p, q)}^{(d, 1)}(\varphi)=\lim _{L \rightarrow \infty}\left[\mathcal{E}_{(p, q)}^{(d, 1)}(\varphi, L)-e_{(p, q)}^{(d, 1)}(L)\right]
$$

Clearly, terms with $\sigma_{i}<1-\frac{d}{2}$ could be added to the sum (2.8) without affecting (2.9). Moreover, when a contribution with $\sigma_{i}=1-\frac{d}{2}$ is present in the sum, one may change the scale of the $\operatorname{logarithm}$, i.e. replace $\log L$ by $\log \mu L$, at the expense of shifting (2.9) by an additive constant $2 \pi c_{i} \log \mu$. Of course, the truncated modular integral $\mathcal{E}_{(p, q)}^{(d, 1)}(L)$ is independent of that choice.

Using known asymptotics of the modular graph functions $E_{a}, D_{4}$, the expansion of the integrands (2.3) at large $\tau_{2}$ is given by

$$
\begin{aligned}
\mathcal{B}_{(1,0)}^{(1)} & =\frac{\pi^{2} \tau_{2}^{2}}{45}+\frac{\zeta(3)}{\pi \tau_{2}}+\mathcal{O}\left(e^{-2 \pi \tau_{2}}\right) \\
\mathcal{B}_{(0,1)}^{(1)} & =\frac{2 \pi^{3} \tau_{2}^{3}}{567}+\frac{\zeta(3)}{3}+\frac{5 \zeta(5)}{4 \pi^{2} \tau_{2}^{2}}+\mathcal{O}\left(e^{-2 \pi \tau_{2}}\right) \\
\mathcal{B}_{(2,0)}^{(1)} & =\frac{8 \pi^{4} \tau_{2}^{4}}{14175}+\frac{4 \pi \tau_{2} \zeta(3)}{45}+\frac{5 \zeta(5)}{6 \pi \tau_{2}}+\frac{\zeta(3)^{2}}{2 \pi^{2} \tau_{2}^{2}}+\frac{\zeta(7)}{2 \pi^{3} \tau_{2}^{3}}+\mathcal{O}\left(e^{-2 \pi \tau_{2}}\right)
\end{aligned}
$$

Thus, we find that the truncated modular integrals are given, up to exponentially suppressed corrections as $L \rightarrow \infty$, by $^{3}$

$$
\begin{aligned}
& \mathcal{E}_{(0,0)}^{(d, 1)}(\varphi, L)=2 \pi \frac{L^{\frac{d-2}{2}}}{\frac{d-2}{2}}+\mathcal{E}_{(0,0)}^{(d, 1)}(\varphi) \\
& \mathcal{E}_{(1,0)}^{(d, 1)}(\varphi, L)=\frac{2 \pi^{3}}{45} \frac{L^{\frac{d+2}{2}}}{\frac{d+2}{2}}+2 \zeta(3) \frac{L^{\frac{d-4}{2}}}{\frac{d-4}{2}}+\mathcal{E}_{(1,0)}^{(d, 1)}(\varphi) \\
& \mathcal{E}_{(0,1)}^{(d, 1)}(\varphi, L)=\frac{4 \pi^{4}}{567} \frac{L^{\frac{d+4}{2}}}{\frac{d+4}{2}}+\frac{2 \pi}{3} \zeta(3) \frac{L^{\frac{d-2}{2}}}{\frac{d-2}{2}}+\frac{5}{2 \pi} \zeta(5) \frac{L^{\frac{d-6}{2}}}{\frac{d-6}{2}}+\mathcal{E}_{(0,1)}^{(d, 1)}(\varphi)
\end{aligned}
$$

\footnotetext{
${ }^{3}$ These results agree for $d=0$ with eq. (3.12), (3.14), (3.17) in [13], up to normalisation factors. For $d=0$, the constant term $\mathcal{E}_{(p, q)}^{(0,1)}$ can be computed using the lemma in [13, section A.2]. For $d \geq$ 1 , differential equations for the renormalized integrals $\mathcal{E}_{(0,0)}^{(d, 1)}(\varphi)$ with respect to the torus moduli were established in [22, 28].
} 


$$
\begin{aligned}
\mathcal{E}_{(2,0)}^{(d, 1)}(\varphi, L)= & \frac{16 \pi^{5}}{14175} \frac{L^{\frac{d+6}{2}}}{\frac{d+6}{2}}+\frac{8 \pi^{2} \zeta(3)}{45} \frac{L^{\frac{d}{2}}}{\frac{d}{2}}+\frac{5 \zeta(5)}{3} \frac{L^{\frac{d-4}{2}}}{\frac{d-4}{2}}+\frac{\zeta(3)^{2}}{\pi} \frac{L^{\frac{d-6}{2}}}{\frac{d-6}{2}} \\
& +\frac{\zeta(7)}{\pi^{2}} \frac{L^{\frac{d-8}{2}}}{\frac{d-8}{2}}+\mathcal{E}_{(2,0)}^{(d, 1)}(\varphi)
\end{aligned}
$$

In these expressions, for a given dimension $d$, terms proportional to $L^{\alpha}$ with $\alpha<0$ should be omitted, while terms of the form $L^{\frac{d-k}{2}} / \frac{d-k}{2}$ for $d=k$ should be understood as $\log L$, as prescribed in (2.8). As we shall explain in section 3, the fact that the coefficients in these expansions involve the same odd zeta values which appear in the tree-level couplings (1.3) is not a concidence.

We note that a different way of regularizing modular integrals of the form (2.1) is to insert a power $\tau_{2}^{\epsilon}$ in the integrand, and take the constant term in the Laurent expansion around $\epsilon=0$. This mimicks the effect of dimensional regularisation prescription in quantum field theory, where one is instructed to compute the loop integral in $D-2 \epsilon$ non-compact dimensions, and take the limit $\epsilon \rightarrow 0$ after subtracting all polar terms. Note that the insertion of $\tau_{2}^{\epsilon}$ breaks modular invariance (but not T-duality), and is only meaningful provided $\mathcal{F}_{1}$ is the standard 'keyhole' fundamental domain, as opposed to one of its modular transforms. This prescription is equivalent for the purpose of computing the coefficient of the logarithmic terms, though the scale of the log would be in general different (see [29] for a recent mathematical discussion of regularized modular integrals).

\subsection{Genus-two}

Similarly, by expanding the integrand of the genus-two four-graviton scattering amplitude computed in [18] in powers of Mandelstam variables at fixed values of the period matrix $\Omega$ in the Siegel upper-half plane $\mathcal{H}_{2}$, one finds a contribution to the effective coupling $\mathcal{E}_{(p, q)}^{(d)}$ of the form $[19,22]$

$$
\mathcal{E}_{(p, q)}^{(d, 2)}(\varphi, L)=\frac{\pi}{4} \int_{\mathcal{F}_{2}(L)} \mathrm{d} \mu_{2} \Gamma_{d, d, 2}(\Omega ; \varphi) \mathcal{B}_{(p, q)}^{(2)}(\Omega),
$$

where $\mathrm{d} \mu_{2}$ is the invariant measure on the Siegel upper-half plane $\mathcal{H}_{2}, \Gamma_{d, d, 2}(\Omega ; \varphi)$ is the genus-two Siegel-Narain theta series

$$
\Gamma_{d, d, 2}(\Omega ; \varphi)=\left|\Omega_{2}\right|^{d / 2} \sum_{\left(m_{i}^{I}, n^{i, I}\right) \in \mathbb{Z}^{2 d}} e^{-\pi \mathcal{L}^{I J} \Omega_{2, I J}+2 \pi \mathrm{i} m_{i}^{I} n^{i, J} \Omega_{1, I J}}
$$

where, similarly to $(2.2)$,

$$
\mathcal{L}^{I J}=\left(m_{i}^{I}+B_{i j} n^{j, I}\right) G^{i k}\left(m_{k}^{J}+B_{k l} n^{l, J}\right)+n^{i, I} G_{i j} n^{j, J} .
$$

The factor $\mathcal{B}_{(p, q)}^{(2)}$ in the integrand is in general a real-analytic Siegel modular function, regular away from the divisor $v=0$ and its images under $\operatorname{Sp}(4, \mathbb{Z})$, and belongs to the class of genus-two modular graph functions of weight $w=2 p+3 q-2$ considered in [21]. For low values of $(p, q)$, it is given explicitly by

$$
\mathcal{B}_{(0,0)}^{(2)}=0, \quad \mathcal{B}_{(1,0)}^{(2)}=2, \quad \mathcal{B}_{(0,1)}^{(2)}=4 \varphi_{\mathrm{KZ}}, \quad \mathcal{B}_{(2,0)}^{(2)}=\frac{1}{2}\left(\mathcal{Z}_{1}-2 \mathcal{Z}_{2}+\mathcal{Z}_{3}\right), \quad \ldots
$$


where $\varphi_{\mathrm{KZ}}$ is the Kawazumi-Zhang invariant $[19,30]$, introduced in the mathematics literature in [31, 32], and $\mathcal{Z}_{a}, a=1,2,3$ are the genus-two string invariants defined in [23, (2.12)] and studied extensively there. Finally,

$$
\mathcal{F}_{2}(L)=\mathcal{F}_{2} \cap\left\{\Omega=\left(\begin{array}{cc}
\tau & v \\
v & \sigma
\end{array}\right): \quad \frac{\operatorname{det} \operatorname{Im} \Omega}{\operatorname{Im} \tau}<L\right\}
$$

is a truncated version $[22]$ of the standard fundamental domain $\mathcal{F}_{2}$ for the action of $\operatorname{Sp}(4, \mathbb{Z})$ on $\mathcal{H}_{2}$, whose interior is defined by the conditions [33] [20, A.15]

$$
\begin{array}{ll}
-\frac{1}{2}<\tau_{1}, v_{1}, \sigma_{1}<\frac{1}{2}, & 0<2 v_{2}<\tau_{2}<\sigma_{2}, \\
|\operatorname{det}(C \Omega+D)|>1, & \text { for all }\left(\begin{array}{cc}
A & B \\
C & D
\end{array}\right) \in \operatorname{Sp}(4, \mathbb{Z}) .
\end{array}
$$

To justify why (2.21) is an appropriate regularization, we first recall some basic facts about the moduli space of genus-two Riemann surfaces.

Boundaries of the moduli space of genus-two Riemann surfaces. The moduli space $\mathcal{M}_{2}$ of genus-two Riemann surfaces without punctures is isomorphic to the complement of the divisor $\Omega_{12}=0$ in the fundamental domain $\mathcal{F}_{2}$. Its compactification, known as the Deligne-Mumford moduli space, is obtained by adding two divisors, corresponding to the separating and non-separating degenerations. In order to discuss these degenerations, it is useful to introduce several (redundant) coordinate systems for $\operatorname{Im} \Omega$,

$$
\operatorname{Im} \Omega=\left(\begin{array}{cc}
L_{1}+L_{2} & L_{1} \\
L_{1} & L_{1}+L_{3}
\end{array}\right)=\left(\begin{array}{cc}
\tau_{2} & \tau_{2} u_{2} \\
\tau_{2} u_{2} & t+\tau_{2} u_{2}^{2}
\end{array}\right)=\frac{1}{V S_{2}}\left(\begin{array}{cc}
1 & S_{1} \\
S_{1} & |S|^{2}
\end{array}\right)
$$

where $L_{1}, L_{2}, L_{3}, t, V$ are real positive variables, $v=u_{1}+\tau u_{2} \in \mathbb{C} /(\mathbb{Z}+\tau \mathbb{Z})$, and $S=S_{1}+\mathrm{i} S_{2}$ lies in the Poincaré upper half-plane. In the standard fundamental domain (2.22), one has $L_{1}<L_{2}<L_{3}$ while $S$ lies in the positive half $\mathcal{F}_{1} \cap\left\{S_{1}>0\right\}$ of the standard fundamental domain $\mathcal{F}_{1}$, which we denote by $\mathcal{F}$. This half-domain is in fact a fundamental domain for the action (B.2) of $\mathrm{GL}(2, \mathbb{Z})$ on $\mathcal{H}_{1}$. In terms of these variables, the relevant degenerations are as follows: ${ }^{4}$

i) The locus $v=0$ (which sits inside $\mathcal{F}_{2}$ but on the boundary of $\mathcal{M}_{2}$ ) corresponds to the separating degeneration limit where the surface $\Sigma_{2}$ degenerates into a pair of elliptic curves joined by a very long tube. Fortunately, the Siegel-Narain partition function (2.18) is regular on this locus, while $\mathcal{B}_{(p, q)}^{(2)}$ grows only as a power $(\log |v|)^{w}[23$, section 4] so that the integral converges in this region.

ii) The 'minimal' non-separating degeneration corresponds to setting $L_{3} \rightarrow \infty$ keeping $L_{1}, L_{2}$ and $\operatorname{Re} \Omega$ fixed; or equivalently $t \rightarrow \infty$ keeping $\tau, v, \sigma_{1}$ fixed. In this limit, $\Sigma_{2}$ degenerates into an elliptic curve $\Sigma_{1}$ of modulus $\tau$, with two marked points at $z=0$ and $z=v$ on $\Sigma_{1}$ (up to an irrelevant translation), and joined by a very long tube of proper length $t$. This region is responsible for one-loop subdivergences.

\footnotetext{
${ }^{4}$ Further degenerations combining separating and non-separating degenerations are allowed but will not be relevant for our purposes.
} 
iii) The 'double' non-separating degeneration corresponds to setting $L_{2}, L_{3} \rightarrow \infty$ at the same rate, keeping $L_{1}$ and $\operatorname{Re} \Omega$ fixed; equivalently $\tau_{2}, \sigma_{2} \rightarrow \infty$ keeping $v$ fixed, or $V, S_{1} \rightarrow 0$ keeping $S_{1} / V$ fixed. In this limit, $\Sigma_{2}$ degenerates into an sphere $\Sigma_{0}$ with four marked points with cross-ratio $v$, joined in pairs by two very long tubes of proper length $\tau_{2}$ and $\sigma_{2}$. It turns out ${ }^{5}$ that for the integrands of interest in this paper, this region does not contribute additional divergent terms beyond those caused by the 'minimal' and 'maximal' non-separating degenerations, discussed above and below.

iv) The 'maximal' or 'complete' non-separating degeneration corresponds to setting $L_{1}$, $L_{2}, L_{3} \rightarrow \infty$ at the same rate, or equivalently $V \rightarrow 0$, keeping $\operatorname{Re} \Omega$ fixed. In this limit, $\Sigma_{2}$ degenerates into two spheres with three marked points on each, joined in pair by three propagators of proper length $L_{i}$ so as to form a 'sunset' graph (see diagram (a) on figure 2). This region is responsible for primitive two-loop divergences.

The regularization (2.21) of the fundamental domain amounts to imposing an upper bound $t<L$ on the length for the long tube in the degeneration ii), and an upper bound $L_{1}<$ $L_{2}<L_{3}<L$ on the proper lengths of the three parameters in degeneration iv). It does not regulate the separating degeneration i), but as already mentioned, there are no divergences coming from this region in the cases of interest in this paper.

The truncated modular integral. To determine the $L$-dependence of the truncated modular integral (2.17), it is easiest to compute its derivative with respect to $L$. Since $L$ only affects the upper bound of the $t$ integral, the $L$-derivative is obtained by evaluating the integrand at $t=L$,

$$
L \partial_{L} \mathcal{E}_{(p, q)}^{(d, 2)}(\varphi, L)=\frac{\pi}{4} \frac{2}{L^{2}} \int_{\left[-\frac{1}{2}, \frac{1}{2}\right]^{3}} \mathrm{~d} u_{1} \mathrm{~d} u_{2} \mathrm{~d} \sigma_{1} \int_{\mathcal{F}_{1}\left(\frac{L}{1-u_{2}^{2}}\right)} \mathrm{d} \mu_{1}(\tau)\left[\Gamma_{d, d, 2} \mathcal{B}_{(p, q)}^{(2)}\right]_{t=L}
$$

The prefactor $2 / L^{2}$ comes from the measure $\mathrm{d} \mu_{2}=4 \frac{\mathrm{d} t}{t^{3}} \mathrm{~d} \mu_{1}(\tau) \mathrm{d} u_{1} \mathrm{~d} u_{2} \mathrm{~d} \sigma_{1}$ and the $\mathbb{Z}_{2}$ symmetry acting on $u_{1}, u_{2}, \sigma_{1}$, while the restriction to the domain $\mathcal{F}_{1}\left(L /\left(1-u_{2}^{2}\right)\right)$ comes from the constraint $\tau_{2}<\sigma_{2}=t+\tau_{2} u_{2}^{2}$ defining the fundamental domain $\mathcal{F}_{2}$. Since $\left|u_{2}\right|<\frac{1}{2}$, one has $\tau_{2}<4 L / 3$ therefore the integral over $\tau$ is finite.

In order to disentangle the dependence on $L$ coming from the integrand and from the integration domain, we decompose ${ }^{6} \mathcal{F}_{1}\left(L /\left(1-u_{2}^{2}\right)\right)=\mathcal{F}_{1}\left(L_{0}\right) \sqcup \mathcal{S}_{1}\left(L_{0}, L\right)$, where $\mathcal{F}_{1}\left(L_{0}\right)$ is the truncated fundamendal domain (2.4), while

$$
\mathcal{S}_{1}\left(L_{0}, L\right)=\left\{\tau \in \mathcal{H}_{1}: L_{0}<\tau_{2}<\frac{L}{1-u_{2}^{2}},-\frac{1}{2}<\tau_{1}<\frac{1}{2}\right\}
$$

Here, $L_{0}$ is an arbitrary separating scale, chosen such that $1 \ll L_{0} \ll L$. For the domain $\mathcal{S}_{1}\left(L_{0}, L\right)$, we use the coordinates $V=1 / \sqrt{t \tau_{2}}, S_{2}=\sqrt{t / \tau_{2}}, S_{1}=u_{2}$, so that the conditions

\footnotetext{
${ }^{5}$ Indeed, the local modular Laurent polynomials $A_{i, j}(S)$ arising in the limit $V \rightarrow 0$ are integrable around $S_{1}=0$, even though they are not smooth on this locus.

${ }^{6}$ The domain $\mathcal{F}_{1}\left(L_{0}\right)$ and $\mathcal{S}_{1}\left(L_{0}, L\right)$ correspond to the boundaries at $t=L$ of regions I and II, in the terminology of $[22,34]$.
} 
defining the domain $\mathcal{S}_{1}\left(L_{0}, L\right)$ translate into

$$
|S|>1, \quad S_{2}<\sqrt{L / L_{0}}, \quad V=\frac{S_{2}}{L} .
$$

Using $\mathrm{d} \mu_{2}=8 V^{2} \mathrm{~d} V \mathrm{~d} \mu(S) \mathrm{d} \rho_{1} \mathrm{~d} v_{1} \mathrm{~d} \sigma_{1}$, with $\mathrm{d} \mu(S)=2 \frac{\mathrm{d} S_{1} \mathrm{~d} S_{2}}{S_{2}^{2}}$, we obtain

$$
\begin{aligned}
L \partial_{L} \mathcal{E}_{(p, q)}^{(d, 2)}(\varphi, L)= & \frac{\pi}{2 L^{2}} \int_{\mathcal{F}_{1}\left(L_{0}\right)} \mathrm{d} \mu_{1}(\tau) \int_{\left[-\frac{1}{2}, \frac{1}{2}\right]^{3}} \mathrm{~d} u_{1} \mathrm{~d} u_{2} \mathrm{~d} \sigma_{1}\left[\Gamma_{d, d, 2} \mathcal{B}_{(p, q)}^{(2)}\right]_{t=L} \\
& +2 \pi \int_{\mathcal{F}\left(\sqrt{L / L_{0}}\right)} \mathrm{d} \mu(S) \int_{\left[-\frac{1}{2}, \frac{1}{2}\right]^{3}} \mathrm{~d} \tau_{1} \mathrm{~d} v_{1} \mathrm{~d} \sigma_{1}\left(\frac{S_{2}}{L}\right)^{3}\left[\Gamma_{d, d, 2} \mathcal{B}_{(p, q)}^{(2)}\right]_{V=S_{2} / L}
\end{aligned}
$$

where $\mathcal{F}\left(\sqrt{L / L_{0}}\right)$ is the truncated fundamental domain for the action (B.2) of $\operatorname{GL}(2, \mathbb{Z})$ on the modulus $S$, namely

$$
\mathcal{F}\left(\sqrt{L / L_{0}}\right)=\left\{S \in \mathcal{H}_{1}:|S|>1, S_{2}<\sqrt{L / L_{0}}, \quad 0<S_{1}<\frac{1}{2}\right\}
$$

For large $t=L$, we can approximate the genus-two Narain partition function $\Gamma_{d, d, 2}$ by $t^{d / 2} \Gamma_{d, d, 1}$ with exponential accuracy. The remaining integral over $u_{1}, u_{2}, \sigma_{1}$ picks up the zeroth Fourier-Jacobi coefficient of $\mathcal{B}_{(p, q)}^{(2)}$. We shall assume that for $t \rightarrow \infty$, this constant term is given, up to exponential corrections, by a finite Laurent series

$$
\int_{\left[-\frac{1}{2}, \frac{1}{2}\right.} \mathrm{d} u_{1} \mathrm{~d} u_{2} \mathrm{~d} \sigma_{1} \mathcal{B}_{(p, q)}^{(2)}(\Omega)=\sum_{i} t^{\sigma_{i}} \hat{\mathcal{B}}_{i}(\tau)+\mathcal{O}\left(e^{-2 \pi t}\right)
$$

where $\hat{\mathcal{B}}_{i}(\tau)$ are modular functions of $\tau$ with polynomial growth as $\tau_{2} \rightarrow \infty$. Indeed, in the cases of interest, $\mathcal{B}_{(p, q)}^{(2)}$ itself has a finite Laurent expansion of the form (2.29), with $\sigma_{i} \in[-w, \ldots, w]$ where $w=2 p+3 q-2$, whose coefficients $\hat{\mathcal{B}}_{i}(\tau, v)$ are generalized modular graph functions of weight $w-\sigma_{i}[21]$.

Similarly, for small $V=S_{2} / L$, we can approximate the Narain partition function $\Gamma_{d, d, 2}$ by $V^{-d}$ with exponential accuracy. The remaining integral over $\tau_{1}, v_{1}, \sigma_{1}$ picks up the zeroth Fourier coefficient of $\mathcal{B}_{(p, q)}^{(2)}$. We shall assume that in the limit $V \rightarrow 0$, his constant term is given, up to exponentially small corrections, by a finite Laurent series,

$$
\int_{\left[-\frac{1}{2}, \frac{1}{2}\right]^{3}} \mathrm{~d} \tau_{1} \mathrm{~d} v_{1} \mathrm{~d} \sigma_{1} \mathcal{B}_{(p, q)}^{(2)}(\Omega)=\sum_{j} V^{\alpha_{j}} \tilde{\mathcal{B}}_{j}(S)+\mathcal{O}\left(e^{-1 / \sqrt{V}}\right)
$$

where $\tilde{\mathcal{B}}_{j}(S)$ are functions ${ }^{7}$ on $\mathcal{H}_{1}$, invariant under the action (B.2) of $\mathrm{GL}(2, \mathbb{Z})$, with polynomial growth as $S_{2} \rightarrow \infty$. Indeed, in the cases of interest, $\mathcal{B}_{(p, q)}^{(2)}$ has a Laurent expansion of the form (2.30) with $\alpha_{i} \in[-w, \ldots, 2 w]$, whose coefficients are modular local Laurent polynomials [21], a class of modular invariant but non smooth functions on the Poincaré upper half plane (see appendix B for a definition and properties of these functions).

\footnotetext{
${ }^{7}$ Note that these functions need not be differentiable on the locus $S_{1}=0$ and its images under GL $(2, \mathbb{Z})$, whenever the integrand $\mathcal{B}_{(p, q)}^{(2)}(\Omega)$ is singular in the separating degeneration.
} 
Under the assumptions (2.29) and (2.30), we easily obtain

$$
\begin{aligned}
L \partial_{L} \mathcal{E}_{(p, q)}^{(d, 2)}(\varphi, L)= & \frac{\pi}{2} \sum_{i} L^{\sigma_{i}+\frac{d}{2}-2} \int_{\mathcal{F}_{1}\left(L_{0}\right)} \mathrm{d} \mu_{1}(\tau) \Gamma_{d, d, 1} \hat{\mathcal{B}}_{i} \\
& +2 \pi \sum_{j} L^{d-\alpha_{j}-3} \int_{\mathcal{F}\left(\sqrt{L / L_{0}}\right)} \mathrm{d} \mu(S) S_{2}^{3+\alpha_{j}-d} \tilde{\mathcal{B}}_{j}
\end{aligned}
$$

Assuming that the zero-mode $\int_{0}^{1} \mathrm{~d} \tau_{1} \hat{\mathcal{B}}_{i}(\tau)$ grows like $\sum_{j} \hat{c}_{i, j} \tau_{2}^{\eta_{i, j}}$ as $\tau_{2} \rightarrow \infty$, and that the zero-mode $\int_{0}^{1 / 2} \mathrm{~d} S_{1} \tilde{\mathcal{B}}_{j}(S)$ grows as $\frac{1}{2} \sum_{i} \tilde{c}_{j, i} S_{2}^{\beta_{j, i}}$ as $S_{2} \rightarrow \infty$, we can replace the integrals of $\mathcal{F}_{1}\left(L_{0}\right)$ and over $\mathcal{F}\left(\sqrt{L / L_{0}}\right)$ by their renormalized values

$$
\begin{gathered}
\int_{\mathcal{F}_{1}\left(L_{0}\right)} \mathrm{d} \mu_{1} \Gamma_{d, d, 1} \hat{\mathcal{B}}_{i}=\int_{\mathcal{F}_{1}} \mathrm{~d} \mu_{1} \Gamma_{d, d, 1} \hat{\mathcal{B}}_{i}+2 \sum_{j} \hat{c}_{i, j} \frac{L_{0}^{\eta_{i, j}+\frac{d}{2}-1}}{\eta_{i, j}+\frac{d}{2}-1}+\ldots \\
\int_{\mathcal{F}\left(\sqrt{\frac{L}{L_{0}}}\right)} \mathrm{d} \mu S_{2}^{3-d-\alpha_{j}} \tilde{\mathcal{B}}_{j}=\int_{\mathcal{F}} \mathrm{d} \mu S_{2}^{3-d-\alpha_{j}} \tilde{\mathcal{B}}_{j}+\sum_{i} \tilde{c}_{j, i} \frac{\left(L / L_{0}\right)^{\frac{1}{2}\left(\alpha_{j}+\beta_{j, i}+2-d\right)}}{\alpha_{j}+\beta_{j, i}+2-d}+\ldots
\end{gathered}
$$

where the dots indicate exponentially suppressed corrections as $L_{0} \gg 1$ and $L \gg L_{0}$, respectively. As a result, we obtain

$$
\begin{aligned}
L \partial_{L} \mathcal{E}_{(p, q)}^{(d, 2)}(\varphi, L)= & \frac{\pi}{2} \sum_{i} L^{\sigma_{i}+\frac{d}{2}-2} \int_{\mathcal{F}_{1}} \mathrm{~d} \mu_{1} \Gamma_{d, d, 1} \hat{\mathcal{B}}_{i}+2 \pi \sum_{j} L^{d-\alpha_{j}-3} \int_{\mathcal{F}} \mathrm{d} \mu S_{2}^{3+\alpha_{j}-d} \tilde{\mathcal{B}}_{j} \\
& +\pi \sum_{i, j} \hat{c}_{i, j} \frac{L^{\sigma_{i}+\frac{d}{2}-2} L_{0}^{\eta_{i, j}+\frac{d}{2}-1}}{\eta_{i, j}+\frac{d}{2}-1}+2 \pi \sum_{j, i} \tilde{c}_{j, i} \frac{L^{d-\alpha_{j}-3}\left(L / L_{0}\right)^{\frac{1}{2}\left(\alpha_{j}+\beta_{j, i}+2-d\right)}}{\alpha_{j}+\beta_{j, i}+2-d}
\end{aligned}
$$

By matching the expansions at $\tau_{2} \rightarrow \infty$ and $S_{2} \rightarrow \infty$, one easily shows that the coefficients $\hat{c}_{i, j}$ and $\tilde{c}_{j, i}$ are equal whenever $\eta_{i, j}=-\alpha_{j}-\sigma_{i}$ and $\beta_{j, i}=\alpha_{j}+2 \sigma_{i}$, so that the terms on the last line cancel. Integrating with respect to $L$, we find that in the limit $L \rightarrow \infty$, $\mathcal{E}_{(p, q)}^{(d, 2)}(\varphi, L)$ grows like

$$
\begin{aligned}
e_{(p, q)}^{(d, 2)}(\varphi, L)= & \frac{\pi}{2} \sum_{\sigma_{i}<2-\frac{d}{2}} \frac{L^{\sigma_{i}+\frac{d}{2}-2}}{\sigma_{i}+\frac{d}{2}-2} \int_{\mathcal{F}_{1}} \mathrm{~d} \mu_{1} \Gamma_{d, d, 1} \hat{\mathcal{B}}_{i}+\frac{\pi}{2} \log L \sum_{\sigma_{i}=2-\frac{d}{2}} \int_{\mathcal{F}_{1}} \mathrm{~d} \mu_{1} \hat{\mathcal{B}}_{i} \Gamma_{d, d, 1} \\
& +2 \pi \sum_{\alpha_{j}<d-3} \frac{L^{d-\alpha_{j}-3}}{d-\alpha_{j}-3} \int_{\mathcal{F}} \mathrm{d} \mu S_{2}^{3+\alpha_{j}-d} \tilde{\mathcal{B}}_{j} \\
& +2 \pi \log L \sum_{\alpha_{j}=d-3} \int_{\mathcal{F}} \mathrm{d} \mu \tilde{\mathcal{B}}_{j}+2 \pi(\log L)^{2} \sum_{\substack{\alpha_{j}=d-3 \\
\beta_{j, i}=-1}} \tilde{c}_{j, i},
\end{aligned}
$$

up to an additive constant (and a constant multiple of $\log L$ when $(\log L)^{2}$ terms are present). The 'renormalized' integral is then defined by subtracting both divergent pieces before taking the limit $L \rightarrow \infty[22,34]$,

$$
\mathcal{E}_{(p, q)}^{(d, 2)}(\varphi)=\lim _{L \rightarrow \infty}\left[\mathcal{E}_{(p, q)}^{(d, 2)}(\varphi, L)-e_{(p, q)}^{(d, 2)}(\varphi, L)\right]
$$


Regulated genus-two effective interactions. We now apply the previous prescription to the genus-two effective couplings (2.17). In the limit $t \rightarrow \infty$, the zero-mode of the integrands (2.20) with respect to $u_{1}, u_{2}, \sigma_{1}$ behave as $[23]^{8}$

$$
\begin{aligned}
\int_{\left[-\frac{1}{2}, \frac{1}{2}\right]^{3}} \mathrm{~d} u_{1} \mathrm{~d} u_{2} \mathrm{~d} \sigma_{1} \mathcal{B}_{(0,1)}^{(2)} & =\frac{2 \pi t}{3}+\frac{5 E_{2}}{\pi t}+\mathcal{O}\left(e^{-2 \pi t}\right) . \\
\int_{\left[-\frac{1}{2}, \frac{1}{2}\right]^{3}} \mathrm{~d} u_{1} \mathrm{~d} u_{2} \mathrm{~d} \sigma_{1} \mathcal{B}_{(2,0)}^{(2)}(\Omega) & =\frac{8 \pi^{2}}{45} t^{2}+\frac{10}{3} E_{2}+\frac{5 E_{3}+3 \zeta(3)}{2 \pi t}+\frac{\hat{\mathcal{B}}_{(2,0)}^{(2)}}{t^{2}}+\mathcal{O}\left(e^{-2 \pi t}\right),
\end{aligned}
$$

where we have not attempted to compute the coefficient $\hat{\mathcal{B}}_{(2,0)}^{(2)}$ of the $\mathcal{O}\left(1 / t^{2}\right)$ term. As we shall see momentarily, the fact that the Laurent coefficients involve the same Eisenstein series $E_{2}, E_{3}$ that appear in the integrands (2.3) of the genus-one amplitudes is not a coincidence. In the limit $V \rightarrow 0$, the zero-modes of $\mathcal{B}_{(p, q)}^{(2)}$ with respect to $\operatorname{Re}(\Omega)$ instead behave as $[23,30]$

$$
\begin{aligned}
\int_{\left[-\frac{1}{2}, \frac{1}{2}\right]^{3}} \mathrm{~d}^{3} \Omega_{1} \mathcal{B}_{(0,1)}^{(2)}= & \frac{10 \pi}{3 V} A_{10}+\frac{5 \zeta(3)}{\pi^{2}} V^{2}+\mathcal{O}\left(e^{-1 / \sqrt{V}}\right) \\
\int_{\left[-\frac{1}{2}, \frac{1}{2}\right]^{3}} \mathrm{~d}^{3} \Omega_{1} \mathcal{B}_{(2,0)}^{(2)}= & \frac{\pi^{2}}{V^{2}}\left[-\frac{26}{189} A_{0,0}+\frac{20}{189} A_{0,2}+\frac{20}{99} A_{1,1}+\frac{64}{45} A_{2,0}\right] \\
& +\frac{V \zeta(3)}{\pi}\left[\frac{9}{5} A_{0,1}+\frac{8}{3} A_{1,0}\right]+\frac{3 \zeta(5)}{2 \pi^{3}} V^{3} A_{0,1}+\beta \frac{\zeta(3)^{2}}{\pi^{4}} V^{4}+\mathcal{O}\left(e^{-1 / \sqrt{V}}\right)
\end{aligned}
$$

where $A_{i, j}(S)$ are the local modular functions defined in appendix $\mathrm{B}$, and the coefficient $\beta$ in the last line of (2.40) was left unevaluated in [23]. ${ }^{9}$ As explained in this reference, the leading term in these expansions reproduces the integrand of the two-loop supergravity amplitude, in terms of the Schwinger parameters $L_{1}, L_{2}, L_{3}$ introduced in (2.23). One of the main aims of this note is to elucidate the origin of the subleading coefficients, proportional to odd zeta values.

Using these asymptotics, we can easily extract the divergent terms in the truncated modular integrals $(2.17){ }^{10}$

$$
\mathcal{E}_{(1,0)}^{(d, 2)}(\varphi, L)=4 \pi \frac{L^{d-3}}{d-3} F_{00}(d-3)+\frac{L^{\frac{d-4}{2}}}{\frac{d-4}{2}} \mathcal{E}_{(0,0)}^{(d, 1)}(\varphi)+\mathcal{E}_{(1,0)}^{(d, 2)}(\varphi)
$$

${ }^{8}$ To obtain these results, we start from eq. (3.16) and (3.22) in [23], and use the identities

$$
\int_{\Sigma_{1}} \kappa_{1}(v) g_{a}(v)=0, \quad \int_{\Sigma_{1}} \kappa_{1}(v) g_{a}(v) g_{b}(v)=E_{a+b}, \quad \int_{\Sigma_{1}} \kappa_{1}(v) D_{a}^{(1)}=0
$$

where $\kappa_{1}(v)=\frac{\mathrm{i}}{2 \tau+2} \mathrm{~d} v \wedge \mathrm{d} \bar{v}$ and $g_{a}(v), D_{a}^{(1)}(v)$ are the Kronecker-Eisenstein series defined in eq. (3.25), (3.29) in loc. cit., along with the identity $D_{3}=E_{3}+\zeta(3)$ from [14, (3.11)].

${ }^{9}$ In the first preprint version of this work, eq. (2.40) was off by a factor of 2 , due to a mistake in [23, (5.18)] which has now been corrected. The coefficient $\beta$ in $(2.40)$ is $\frac{\beta+11}{16}$ in [23].

${ }^{10}$ For $d=0$, the modular integral $\mathcal{E}_{(0,1)}^{(d, 2)}(\varphi)$ was computed in [20] using the Laplace eigenvalue property of $\varphi_{\mathrm{KZ}}$. For $d>0$, differential equations for the renormalized integral $\mathcal{E}_{(0,1)}^{(d, 2)}(\varphi)$ were established in $[22,35]$. It would be very interesting to establish similar results for higher derivative interactions. 


$$
\begin{aligned}
& \mathcal{E}_{(0,1)}^{(d, 2)}(\varphi, L)=\frac{20 \pi^{2}}{3} \frac{L^{d-2}}{d-2} F_{10}(d-2)+\frac{10 \zeta(3)}{\pi} \frac{L^{d-5}}{d-5} F_{00}(d-5) \\
& +\frac{\pi}{3} \frac{L^{\frac{d-2}{2}}}{\frac{d-2}{2}} \mathcal{E}_{(0,0)}^{(d, 1)}(\varphi)+\frac{5}{2 \pi} \frac{L^{\frac{d-6}{2}}}{\frac{d-6}{2}} \mathcal{E}_{(1,0)}^{(d, 1)}(\varphi)+\mathcal{E}_{(0,1)}^{(d, 2)}(\varphi) \\
& \mathcal{E}_{(2,0)}^{(d, 2)}(\varphi, L)=2 \pi^{3} \frac{L^{d-1}}{d-1}\left[-\frac{26}{189} F_{00}(d-1)+\frac{20}{189} F_{02}(d-1)+\frac{20}{99} F_{11}(d-1)+\frac{64}{45} F_{20}(d-1)\right] \\
& +2 \zeta(3) \frac{L^{d-4}}{d-4}\left[\frac{9}{5} F_{01}(d-4)+\frac{8}{3} F_{10}(d-4)\right]+\frac{3 \zeta(5)}{\pi^{2}} \frac{L^{d-6}}{d-6} F_{01}(d-6) \\
& +\frac{2 \beta \zeta(3)^{2}}{\pi^{2}} \frac{L^{d-7}}{d-7} F_{00}(d-7)+\frac{4 \pi^{2}}{45} \frac{L^{\frac{d}{2}}}{\frac{d}{2}} \mathcal{E}_{(0,0)}^{(d, 1)}(\varphi)+\frac{5}{3} \frac{L^{\frac{d-4}{2}}}{\frac{d-4}{2}} \mathcal{E}_{(1,0)}^{(d, 1)}(\varphi) \\
& +\frac{1}{4 \pi} \frac{L^{\frac{d-6}{2}}}{\frac{d-6}{2}}\left[3 \mathcal{E}_{(0,1)}^{(d, 1)}+2 \zeta(3) \mathcal{E}_{(0,0)}^{(d, 1)}(\varphi)\right]+\frac{\pi}{2} \frac{L^{\frac{d-8}{2}}}{\frac{d-8}{2}} \int_{\mathcal{F}_{1}} \mathrm{~d} \mu_{1} \hat{\mathcal{B}}_{(2,0)}^{(2)}+\mathcal{E}_{(2,0)}^{(d, 2)}(\varphi)
\end{aligned}
$$

where $F_{i j}(s)$ denotes the integral of $A_{i, j}$ over the fundamental domain of $\operatorname{GL}(2, \mathbb{Z})$,

$$
F_{i j}(s)=\int_{\mathcal{F}} \mathrm{d} \mu(S) S_{2}^{-s} A_{i, j}(S) \text {. }
$$

As discussed in appendix B, this integral converges when $\operatorname{Re}(s)>i+j-1$, and extends to a meromorphic function in the whole $s$-plane, with poles at integers in the range $[-3 i-j-1, \ldots, i+j-1]$. As usual, the factors of $L^{d-k} /(d-k)$ when $d=k$ must be treated by replacing $d$ by $d+2 \epsilon$ and taking the limit $\epsilon \rightarrow 0$ after dropping polar terms, keeping in mind that additional poles can come from the integral $F_{i j}(d-k)$. Again, the fact that the coefficients in these expansions involve the same odd zeta values and (renormalized) modular integrals which occur at tree-level (1.3) and genus-one (2.1), (2.3) is not a coincidence, and will be explained in section 3 .

\subsection{Genus three}

Although the main emphasis of this note is on the genus one and two contributions, it will be useful to recall the leading term in the genus three amplitude, computed using the pure spinor formalism [36]. The genus three amplitude contributes first at order $\nabla^{6} \mathcal{R}^{4}$ in the derivative expansion, with a coefficient given by [37]

$$
\mathcal{E}_{(0,1)}^{(d, 3)}(\varphi, L)=\frac{5}{16} \int_{\mathcal{F}_{3}(L)} \mathrm{d} \mu_{3} \Gamma_{d, d, 3} .
$$

Using the truncated fundamental domain $\mathcal{F}_{3}(L)$ defined in [34], and specializing to the dimensions where logarithmic terms arise, we get (eq. (4.72) in [34], noting that the measure $\mathrm{d} \mu_{h}$ used in that reference differs from the one used here by a factor $1 / 2^{h(h+1) / 2}$ )

$$
\begin{aligned}
\mathcal{E}_{(0,1)}^{(d, 3)}(\varphi, L)= & \mathcal{E}_{(0,1)}^{(d, 3)}(\varphi)+\frac{5}{4} \log L \delta_{d, 6} \int_{\mathcal{F}_{2}} \mathrm{~d} \mu_{2} \Gamma_{6,6,2} \\
& +\frac{5 \pi}{3} \log L \delta_{d, 5} \int_{\mathcal{F}_{2}} \mathrm{~d} \mu_{1} \Gamma_{5,5,1}+5 \zeta(3) \log L \delta_{d, 4} \\
= & \mathcal{E}_{(1,0)}^{(d, 3)}(\varphi)+\frac{5}{2 \pi} \mathcal{E}_{(1,0)}^{(6,2)}(\varphi) \log L \delta_{d, 6} \\
& +\frac{5}{3} \mathcal{E}_{(0,0)}^{(5,1)}(\varphi) \log L \delta_{d, 5}+5 \zeta(3) \log L \delta_{d, 4}
\end{aligned}
$$


The second term in the last line comes from a one-loop subdivergence in $D=4$, where the other two loops shrink to give a $\nabla^{4} \mathcal{R}^{4}$ vertex. The third term comes from a two-loop subdivergence in $D=5$, where the remaining loop shrinks to give a $\mathcal{R}^{4}$ vertex. The last term comes from the primitive three-loop divergence in $D=6$, and is proportional to the volume of the fundamental domain of $\operatorname{PGL}(3, \mathbb{Z})$, given by ${ }^{11} \zeta(3) / 24$.

Unfortunately, the genus-three contributions to higher derivative interactions $\mathcal{E}_{(p, q)}^{(d, 3)}$ with $2 p+3 q>3$ are not known at present, due to difficulties in regulating the integral over the pure spinor ghosts [37]. However, by requiring that coefficients of divergences recombine into U-duality invariant combinations, we shall see that the next-to-leading term must produce the following divergences in $D=6$ and $D=4$,

$$
\begin{aligned}
\mathcal{E}_{(2,0)}^{(d, 3)}(\varphi, L)= & {\left[\frac{5}{3} \mathcal{E}_{(1,0)}^{(4,2)} \log L+\frac{7}{6} \mathcal{E}_{(1,0)}^{(4,2)}(\log L)^{2}\right] \delta_{d, 4} } \\
& +\left[\frac{1}{4 \pi}\left(3 \mathcal{E}_{(0,1)}^{(6,2)}+\frac{1}{2}\left(\mathcal{E}_{(0,0)}^{(6,1)}\right)^{2}\right) \log L+\frac{3}{2 \pi^{2}} \mathcal{E}_{(1,0)}^{(6,1)}(\log L)^{2}\right] \delta_{d, 6}+\ldots
\end{aligned}
$$

In particular, the integrand $\mathcal{B}_{(2,0)}^{(3)}$ must produce the genus-two Kawazumi-Zhang invariant $\varphi_{K Z}$ in the limit where the genus-three curve degenerates into a genus-two curve with two marked points joined by a long tube.

\section{Matching with supergravity divergences}

The appearance of odd zeta values $\zeta(3), \zeta(5), \zeta(3)^{2}, \ldots$ in the asymptotics of the genus one and two integrands, as well as in the resulting truncated modular integrals, strongly suggests that these terms should be related to the tree-level $\mathcal{R}^{4}, \nabla^{4} \mathcal{R}^{4}, \nabla^{6} \mathcal{R}^{4}$ interactions whose coefficients (1.3) involve the same transcendental numbers [23]. In this section, we argue that these terms are indeed related, by matching the logarithmic terms in the truncated modular integrals with ultraviolet divergences of loop amplitudes in supergravity supplemented with higher derivative vertices.

\subsection{Qualitative analysis of UV divergences in supergravity}

Although supergravity amplitudes are most efficiently studied using dimensional regularization, it is instructive to discuss their divergences using a hard UV cut-off $|k|<\Lambda$ on the Euclidean momenta of the particles running in the loop (assuming that such a cutoff is consistent with supersymmetry). We are mostly interested in dimensions $D$ where logarithmic divergences occur, which corresponds to appearance of poles in dimensional regularization.

One-loop. By dimensional analysis, the box diagram $(a)$ in figure 1 diverges in dimension $D$ like

$$
\int \mathrm{d}^{D} k \frac{\left(k^{2}\right)^{4}}{\left(k^{2}\right)^{4}} \sim \Lambda^{D-8} \mathcal{R}^{4}+\Lambda^{D-12} \nabla^{4} \mathcal{R}^{4}+\Lambda^{D-14} \nabla^{6} \mathcal{R}^{4}+\Lambda^{D-16} \nabla^{8} \mathcal{R}^{4}+\ldots
$$

\footnotetext{
${ }^{11}$ As far as I know, $\zeta(3)$ here is completely unrelated to the coefficient of the tree-level $\mathcal{R}^{4}$ interaction!
} 


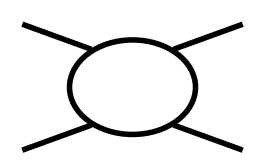

(a)

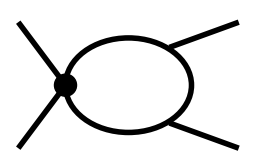

(b)

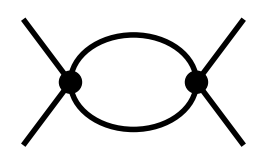

$(c)$

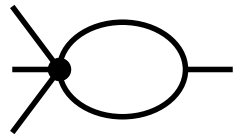

$(d)$

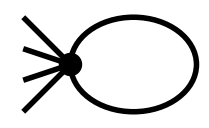

$(e)$

Figure 1. (a) Box diagram in supergravity; (b) Triangle diagram with one $\nabla^{4 p+6 q} \mathcal{R}^{4}$ quartic vertex; $(c)$ Bubble diagram with two quartic vertices; $(d)$ Bubble diagram with one quintic and one cubic vertex; $(e)$ Tadpole diagram with one sextic vertex.

where we used the fact that the leading term in the low energy expansion involves eight powers of momenta, which combine with the eight polarization tensors into a $\mathcal{R}^{4}$ vertex, while the next-to-leading correction $\nabla^{2} \mathcal{R}^{4}$ vanishes on-shell. Upon identifying $\Lambda=1 /\left(\ell_{s} \sqrt{L}\right)$, we observe that exactly the same powers of $L$ appear in the leading term in the expansion of the genus-one truncated modular integrals (2.13)-(A.3). When $D=8$, the coefficient of $\mathcal{R}^{4}$ becomes proportional to $\log \Lambda$, in agreement with the $\log L$ term in (2.13) for $d=2$. The higher-derivative terms in (3.1) give logarithmic divergences in $D \geq 12$, but those are unphysical since they occur above the critical dimension $D=10$.

However, in the presence of higher-derivative tree-level vertices in the action, of the form $\int \mathrm{d}^{D} x \sqrt{-g} \mathcal{E}_{(p, q)}^{(d, 0)} \nabla^{4 p+6 q}(\varphi, L) \mathcal{R}^{4}$, the one-loop amplitude also includes a triangle graph $(b)$ with one quartic vertex, and a bubble graph $(c)$ with two quartic vertices. For $(p, q)=(0,0)$, the triangle graph $(b)$ leads to divergences of the form ${ }^{12}$

$$
\mathcal{E}_{(0,0)}^{(d, 0)} \int \mathrm{d}^{D} k \frac{\left(k^{2}\right)^{2} k^{8}}{\left(k^{2}\right)^{3}} \sim \mathcal{E}_{(0,0)}^{(d, 0)}\left[\Lambda^{D-2} \mathcal{R}^{4}+\Lambda^{D-6} \nabla^{4} \mathcal{R}^{4}+\Lambda^{D-8} \nabla^{6} \mathcal{R}^{4}+\Lambda^{D-10} \nabla^{8} \mathcal{R}^{4}+\ldots\right]
$$

The term proportional to $\Lambda^{D-6}, \Lambda^{D-8}, \Lambda^{D-10}$ lead to a logarithmic divergence in $D=$ $6,8,10$, corresponding to the second term in (2.14), (2.15) and (2.16). It is important to stress that in (3.2), we used the on-shell value of the quartic vertex, whereas two legs are a priori off-shell. This does not affect the imaginary part of the scattering amplitude in the forward limit, and therefore should preserve the structure of the UV divergences, as we further elaborate in section 4 .

Similarly, the triangle graph $(b)$ with one $\mathcal{E}_{(1,0)}^{(d, 0)}(\varphi, L) \nabla^{4} \mathcal{R}^{4}$ vertex produces

$$
\mathcal{E}_{(1,0)}^{(d, 0)} \int \mathrm{d}^{D} k \frac{\left(k^{2}\right)^{2} k^{12}}{\left(k^{2}\right)^{3}} \sim \mathcal{E}_{(1,0)}^{(d, 0)}\left[\Lambda^{D+2} \mathcal{R}^{4}+\Lambda^{D-2} \nabla^{4} \mathcal{R}^{4}+\Lambda^{D-4} \nabla^{6} \mathcal{R}^{4}+\Lambda^{D-6} \nabla^{8} \mathcal{R}^{4}+\ldots\right]
$$

The terms proportional to $\Lambda^{D-4}, \Lambda^{D-6}, \Lambda^{D-8} \ldots$ lead to logarithmic divergences in $D=$ $4,6,8, \ldots$, correspond to the third term in $(2.15),(2.16)$, and (A.3). The term proportional

\footnotetext{
${ }^{12}$ This can be viewed as the form factor of the supersymmetric completion of the operator $\mathcal{R}^{4}$ at one loop and zero momentum, as emphasized in [38].
} 
to $\Lambda^{D-2}$ indicates a divergence proportional to $\nabla^{4} \mathcal{R}^{4}$ in $D=2$, but its physical significance is unclear due to the usual difficulties in defining graviton scattering in $D=2$.

In turn, the triangle graph (b) with one $\nabla^{6} \mathcal{R}^{4}$ vertex, along with the bubble graph (c) with two $\mathcal{R}^{4}$ vertices, produce divergences of the form

$$
\left[\mathcal{E}_{(0,1)}^{(d, 0)}+\left(\mathcal{E}_{(0,0)}^{(d, 0)}\right)^{2}\right]\left[\Lambda^{D+4} \mathcal{R}^{4}+\Lambda^{D} \nabla^{4} \mathcal{R}^{4}+\Lambda^{D-2} \nabla^{6} \mathcal{R}^{4}+\Lambda^{D-4} \nabla^{8} \mathcal{R}^{4}+\Lambda^{D-6} \nabla^{10} \mathcal{R}^{4}+\ldots\right]
$$

where the relative coefficient between $\mathcal{E}_{(0,1)}^{(d, 0)}$ and $\left(\mathcal{E}_{(0,0)}^{(d, 0)}\right)^{2}$ is not known. The term proportional to $\Lambda^{D-4}$ correspond to the fourth term in (2.16), while the term proportional to $\Lambda^{D-6}$ correspond to the fourth term in (A.3).

In addition, the tree-level action in string theory contains higher order gravitational vertices of the form $\nabla^{2 n} \mathcal{R}^{5}$ with $n \geq 1, \nabla^{2 n} \mathcal{R}^{6}$ with $n \geq 0$, etc, [39, 40], which can be used to construct the bubble graph $(d)$ with one quintic vertex, or the tadpole graph $(e)$ with one cubic vertex. The latter has no dependence on the external momenta and can be safely ignored. As for the contributions of type $(d)$, at tree level they are related by non-linear supersymmetry to $\nabla^{4 p+6 q} \mathcal{R}^{4}$ couplings with $4 p+6 q=2 n-2$, in which case they produce the same type of divergences as (3.3), (3.4), etc. Therefore there are no additional contributions at first order in the genus expansion.

Two-loop. We now turn to the two-loop supergravity amplitude. By dimensional analysis, the two-loop sunset graph $(a)$ in figure 2 diverge like

$$
\int \mathrm{d}^{2 D} k \frac{\left(k^{2}\right)^{6}}{\left(k^{2}\right)^{7}} \sim \Lambda^{2 D-14} \nabla^{4} \mathcal{R}^{4}+\Lambda^{2 D-16} \nabla^{6} \mathcal{R}^{4}+\Lambda^{2 D-18} \nabla^{8} \mathcal{R}^{4}+\ldots
$$

where we took into account the fact that the leading term in the low energy expansion starts at order $\nabla^{4} \mathcal{R}^{4}[25]$. After substituting $\Lambda=1 / \sqrt{\alpha^{\prime} L}$, we see that the same powers of $L$ appear in the first term in the expansions (2.41)-(2.43). These terms generate logarithmic divergences in $D=7,8,9, \ldots$, corresponding to the terms proportional to $L^{d-3}$ in (2.41), $L^{d-2}$ in (2.42) and $L^{d-1}$ in (2.43), respectively. In addition to the primitive divergence (3.5), there are also one-loop subdivergences, obtained by inserting one of the one-loop supergravity divergences in (3.1) at the quartic vertex of a triangle diagram $(b)$ in figure 1. The resulting divergences are similar to (3.2)-(3.4), upon replacing the treelevel coefficient $\mathcal{E}_{(1,0)}^{(d, 0)}$ is replaced by the divergent part of the one-loop coefficient $\mathcal{E}_{(1,0)}^{(d, 1)}(L)$. In dimension $D=8$, this leads to a $(\log \Lambda)^{2}$ divergence, or a $1 / \epsilon^{2}$ pole in dimensional regularization, in agreement with the supergravity computation in [25] (see (3.16) below).

In addition, the diagrams $(b, c)$ in figure 2 , where two of the supergravity cubic vertices are replaced by a tree-level $\mathcal{R}^{4}$ vertex give divergences of the form

$$
\mathcal{E}_{(0,0)}^{(d, 0)} \int \mathrm{d}^{2 D} k \frac{\left(k^{2}\right)^{4} k^{8}}{\left(k^{2}\right)^{6}} \sim \mathcal{E}_{(0,0)}^{(d, 0)}\left[\Lambda^{2 D-8} \nabla^{4} \mathcal{R}^{4}+\Lambda^{2 D-10} \nabla^{6} \mathcal{R}^{4}+\Lambda^{2 D-12} \nabla^{8} \mathcal{R}^{4}+\ldots\right]
$$

The second and third terms generate logarithmic singularities in $D=5$ and $D=6$, corresponding to the terms proportional to $\zeta(3) \Lambda^{d-5}$ in (2.42) and to $\zeta(3) \Lambda^{d-4}$ in (2.43). The first term in (3.6) would generate a logarithmic divergence proportional to $\nabla^{4} \mathcal{R}^{4}$ in 


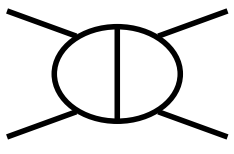

(a)

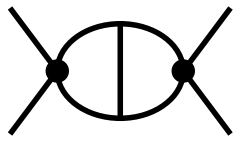

(d)

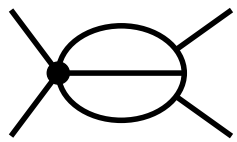

$(g)$

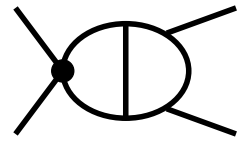

(b)

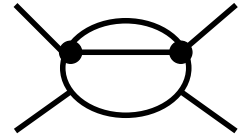

$(e)$

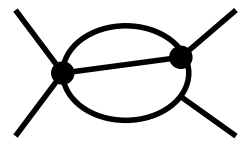

(h)

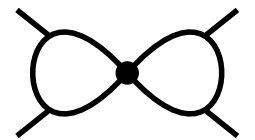

(c)

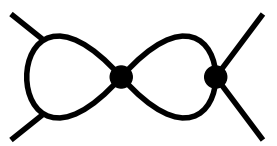

$(f)$

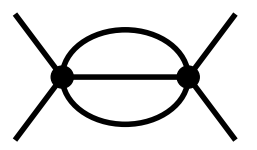

$(j)$

Figure 2. (a) Two-loop 'sunset' diagram in supergravity (drawn sideways); (b,c) Two-loop diagrams with one quartic vertex; $(d, e, f)$ Two-loop diagrams with two quartic vertices; $(g, h, j)$ Two-loop diagrams with quintic vertices; variants of these diagrams with non-planar topologies are also allowed.

$D=4$, but no such divergence appears in (2.41), so such a term is presumably ruled out by supersymmetry. ${ }^{13}$ Similarly, if two of the supergravity cubic vertices are replaced by a tree-level $\nabla^{4} \mathcal{R}^{4}$ vertex, we get

$$
\mathcal{E}_{(1,0)}^{(d, 0)} \int \mathrm{d}^{2 D} k \frac{\left(k^{2}\right)^{4} k^{12}}{\left(k^{2}\right)^{6}} \sim \mathcal{E}_{(1,0)}^{(d, 0)}\left[\Lambda^{2 D-4} \nabla^{4} \mathcal{R}^{4}+\Lambda^{2 D-6} \nabla^{6} \mathcal{R}^{4}+\Lambda^{2 D-8} \nabla^{8} \mathcal{R}^{4}+\ldots\right]
$$

The term proportional to $\Lambda^{2 D-8}$ generates a logarithmic divergence in $D=4$, corresponding to the term proportional to $\zeta(5) L^{d-6}$ in (2.43), while the term proportional to $\Lambda^{2 D-6}$ suggests a logarithmic divergence proportional to $\nabla^{6} \mathcal{R}^{4}$ in $D=3$, which is however not present in (2.42). Again, such a term is presumably ruled out by supersymmetry, since the coefficients $\mathcal{E}_{(1,0)}^{(d)}$ and $\mathcal{E}_{(0,1)}^{(d)}$ for $d=7$ have different eigenvalues under the Laplace operator on $\mathcal{M}_{D}$.

Finally, replacing two of the supergravity vertices by a $\nabla^{6} \mathcal{R}^{4}$ vertex (still corresponding to the diagrams $(b, c)$ in figure 2 ), or four supergravity vertices by two $\mathcal{R}^{4}$ vertices (corresponding to the diagrams $(d, e, f)$ in figure 2), we get in either case

$$
\left[\mathcal{E}_{(0,1)}^{(d, 0)}+\left(\mathcal{E}_{(0,0)}^{(d, 0)}\right)^{2}\right]\left[\Lambda^{2 D-2} \nabla^{4} \mathcal{R}^{4}+\Lambda^{2 D-4} \nabla^{6} \mathcal{R}^{4}+\Lambda^{2 D-6} \nabla^{8} \mathcal{R}^{4}+\ldots\right]
$$

\footnotetext{
${ }^{13}$ Indeed, the coefficients $\mathcal{E}_{(0,0)}^{(d)}$ and $\mathcal{E}_{(1,0)}^{(d)}$ have different eigenvalues under the Laplace operator on $\mathcal{M}_{D}$, so cannot mix. I am grateful to G. Bossard for pointing this out.
} 


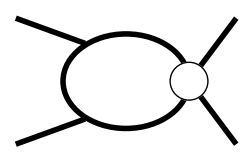

$(k)$

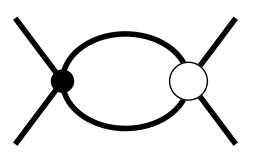

$(l)$

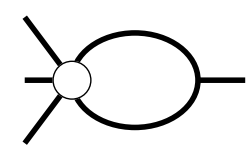

$(m)$

Figure 3. One-loop diagrams with genus-one quartic or quintic vertex (denoted by a white circle).

The term proportional to $\Lambda^{2 D-6}$ generates a logarithmic singularity in $D=3$ corresponding to the term proportional to $\zeta(3)^{2} L^{d-7}$ in (2.43).

Still, this does not exhaust the set of two-loop diagrams which occur in the low energy limit of string theory. First, in addition to the tree-level $\nabla^{4 p+6 q} \mathcal{R}^{4}$ quartic vertices, the treelevel quintic vertices of the form $\nabla^{2 n} \mathcal{R}^{5}$ with $n \geq 1$ can be used to construct the two-loop diagrams $(g, h, j)$ on figure 2 . Power counting shows that the diagrams $(h, j)$ superficially diverge as $\Lambda^{2 D+14}$ and $\Lambda^{2 D+18}$ or faster, so do not lead to logarithmic divergences in dimension $D \geq 3$ up to order $\nabla^{10} \mathcal{R}^{4}$ included. In contrast, the diagram $(g)$ diverges as $\Lambda^{2 D+2 n-6} \nabla^{4} \mathcal{R}^{4}$; this is the same scaling as (3.7) or (3.8) for $n=1$ or $n=2$, in agreement with the fact that the $\nabla^{2 n} \mathcal{R}^{5}$ tree-level coupling is related by non-linear supersymmetry to the $\nabla^{2 n-2} \mathcal{R}^{4}$ couplings. Thus there are no additional divergences from diagrams $(g, h, j)$.

More importantly however, there are also triangle and bubble diagrams with an insertion of genus-one vertices $\mathcal{E}_{(p, q)}^{(d, 1)} \nabla^{4 p+6 q} \mathcal{R}^{4}$ (see figure 3 ). The diagrams $(k, l)$ can be analyzed in the same way as the one-loop diagrams in the previous subsection, upon replacing $\mathcal{E}_{(p, q)}^{(d, 0)}$ by $\mathcal{E}_{(p, q)}^{(d, 1)}$ in $(3.2)-(3.4)$. It is easy to check that they reproduce the one-loop divergences of the form $\Lambda^{(d-k) / 2} \mathcal{E}_{(p, q)}^{(d, 1)} / \frac{d-k}{2}$ in $(2.41)-(2.43)$. In fact, combining this supergravity description with U-duality, we predict that these one-loop subdivergences must combine with the divergences in (2.14)-(2.16) of the genus one couplings so as to form a multiple of the U-duality invariant coefficient $\mathcal{E}_{(p, q)}^{(d)} \propto g_{D}^{-2} \mathcal{E}_{(p, q)}^{(d, 0)}+\mathcal{E}_{(p, q)}^{(d, 1)}+\ldots$ Expanding this at the next order in $g_{D}$ also predicts the form of the one-loop and two-loop subdivergences in the genus-three amplitude, as we discuss below. We verify some of these predictions in subsection 3.2.

As for the diagram $(m)$ in figure 3 , it leads to the same type of divergences as the diagram $(d)$ in figure 1 , with the important difference that at one-loop, some of the quintic vertices of the form $\nabla^{2 n} \mathcal{R}^{5}$ with $n \geq 3$ are no longer related by non-linear supersymmetry to $\nabla^{4 p+6 q} \mathcal{R}^{4}$ couplings [41]. For $n=3$, we find a superficial divergence of order $\Lambda^{D+14}$, which may lead to a logarithmic divergence at order $\nabla^{10} \mathcal{R}^{4}$ in $D=4, \nabla^{12} \mathcal{R}^{4}$ in $D=6$, etc, with a new set of U-duality invariant coefficients. Since we mostly restrict to order $\nabla^{8} \mathcal{R}^{4}$ in this note, we can ignore such contributions.

Three-loop. Finally, let us briefly consider three-loop diagrams. In supergravity, they occur into two topologies, the tetrahedron (or 'Mercedes') and ladder diagrams (see figure 4). Under naive power counting, both lead to the same structure of primitive divergences,

$$
\int \mathrm{d}^{3 D} k \frac{\left(k^{2}\right)^{8}}{\left(k^{2}\right)^{10}} \sim \Lambda^{3 D-18} \nabla^{6} \mathcal{R}^{4}+\Lambda^{3 D-20} \nabla^{8} \mathcal{R}^{4}+\Lambda^{3 D-22} \nabla^{10} \mathcal{R}^{4}+\ldots
$$




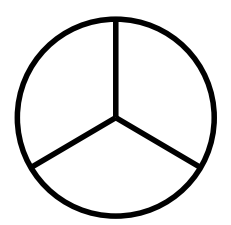

(a)

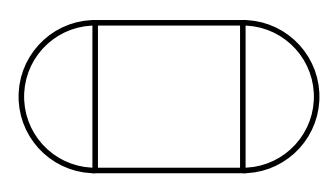

(b)

Figure 4. (a) Skeleton of the tetrahedron diagram; (b) Skeleton of the three-loop ladder diagram; in both cases, the four external gravitons can attach to any of the edges.

where we took into account the fact that the leading term in the derivative expansion starts at order $\nabla^{6} \mathcal{R}^{4}$ (and in fact comes only from the tetrahedron diagram) [26]. The first term in (3.21) produces a logarithmic divergence in $D=6$, in agreement with the three-loop divergence computed in [26] using dimensional regularisation. In addition, there are also one-loop subdivergences, obtained by inserting one of the two-loop divergent terms in (3.5) at the quartic vertex of a triangle diagram $(b)$ in figure 1 ; these contributions take the same form as (3.2)-(3.4), upon replacing the tree-level coefficient $\mathcal{E}_{(1,0)}^{(d, 0)}$ by the divergent part of the one-loop term $\mathcal{E}_{(1,0)}^{(d, 1)}(L)$. Finally, there are also two-loop subdivergences, obtained by inserting one of the one-loop divergences in (3.1) at the quartic vertices of the diagrams $(b, c)$ in figure 2 ; they take the same form as (3.6)-(3.8), upon replacing $\mathcal{E}_{(1,0)}^{(d, 0)}$ by the divergent part of the two-loop term $\mathcal{E}_{(1,0)}^{(d, 2)}(L)$. For $D=8,10$, this produces $(\log \Lambda)^{2}$ and $(\log \Lambda)^{3}$ terms, albeit at high order in the derivative expansion $\left(\nabla^{12} \mathcal{R}^{4}\right.$ and $\nabla^{18} \mathcal{R}^{4}$, respectively).

Inserting higher derivative vertices, we get additional $\log$ divergences in $D=4$ and $D=2$ (and in fractional dimensions which we ignore):

$$
\mathcal{E}_{(0,0)}^{(d)} \Lambda^{3 D-12} \nabla^{6} \mathcal{R}^{4}+\mathcal{E}_{(1,0)}^{(d)} \Lambda^{3 D-12} \nabla^{10} \mathcal{R}^{4}+\left[\mathcal{E}_{(0,1)}^{(d)}+\left(\mathcal{E}_{(0,0)}^{(d)}\right)^{2}\right] \Lambda^{3 D-6} \nabla^{6} \mathcal{R}^{4}+\ldots
$$

Again, the logarithmic divergence proportional to $\nabla^{6} \mathcal{R}^{4}$ in $D=4$ does not match any pole in (3.23), so its coefficient presumably vanishes.

\subsection{Combining genus-one and genus-two scale-dependent terms}

As explained above, dimensional analysis in supergravity supplemented with higherderivative stringy vertices gives a simple prediction for the dimensions $d$ where the truncated modular integrals can have logarithmic divergences, and for the coefficients of these logarithmic divergences. In particular, it requires that the coefficients of the genus-one divergence and of the one-loop subdivergence of the genus-two divergence must recombine into the leading terms in the U-duality invariant coupling $\mathcal{E}_{(p, q)}^{(d)}$. In this subsection, we consider each dimension in turn, and check that these predictions are indeed verified.

- In $D=10$, the logarithms coming from the divergence (2.16) of the genus-one amplitude and the one-loop subdivergence (2.43) of the genus-two amplitude combine into

$$
\mathcal{E}_{(2,0)}^{(0)}(\varphi, L) \sim g_{s}^{1 / 2}\left[\frac{8 \pi^{2} \zeta(3)}{45} g_{s}^{-2}+\frac{4 \pi^{2}}{45} \mathcal{E}_{(0,0)}^{(0,1)}+\ldots\right] \log L=\frac{4 \pi^{2}}{45} \mathcal{E}_{(0,0)}^{(0)} \log L
$$


Here, the overall power of $g_{D}$ comes from rescaling from string to Einstein frame, and the dots indicate divergent contributions from the instanton series, which are predicted by U-duality. As noted below (3.2), The contribution $\mathcal{E}_{(0,0)}^{(0)} \log L$ can be understood as the divergence of a triangle diagram in supergravity with one $\mathcal{R}^{4}$ quartic vertex involving both tree-level and one-loop contribution. While it would be desirable to recover the coefficient $\frac{4 \pi^{2}}{45}$ by an independent computation of the divergence of the one-loop form factor in supergravity, this coefficient follows here from a straightforward analysis of the subdivergence of the genus-two string amplitude. At order $\nabla^{10} \mathcal{R}^{4}$, there is also a two-loop supergravity divergence in $D=10$, as discussed in appendix A.

- In $D=9$, there is a single divergent term up to order $\nabla^{8} \mathcal{R}^{4}$, from (2.43). Its coefficient is easily computed using (B.16),

$$
\mathcal{E}_{(2,0)}^{(1)}(\varphi, L) \sim-\frac{52 \pi^{4}}{567} \log L
$$

According to the discussion below (3.5), this should come from the two-loop divergence (3.13) in supergravity. In order to check the numerical factor, we quote the result of the two-loop supergravity divergence in $D=9[25,(4.3)]$

$$
\mathcal{I}_{2}^{D=9-2 \epsilon} \sim-\left(\frac{\kappa}{2}\right)^{6} \frac{13 \pi}{9072} \frac{1}{4 \epsilon(4 \pi)^{9}}\left(s^{2}+t^{2}+u^{2}\right)^{2} s t u M_{4}^{\text {tree }}
$$

To translate to our conventions, recall that $\sigma_{n}=\left(s^{n}+t^{n}+u^{n}\right) \ell_{D}^{2 n} / 4^{n}$, while the Newton constant $\kappa^{2}$ is related to the Planck length $\ell_{D}$ in dimension $D$ by ${ }^{14} \kappa^{2}=$ $2(2 \pi)^{D-3} \ell_{D}^{D-2}$. Identifying $s t u M_{4}^{\text {tree }}=64 \kappa^{2} \mathcal{R}^{4}$, and recalling that in dimensional regularization, a polar term at $h$ loop comes with a coefficient of the form $\frac{L^{h \epsilon}}{\epsilon}=$ $\frac{1}{\epsilon}+h \log L+\ldots$ so a pole $1 / \epsilon$ corresponds to a $\log$ arithmic term $h \log L$, we get

$$
\mathcal{I}_{2}^{D=9-2 \epsilon} \sim-\frac{52 \pi^{4}}{567} \log L \frac{\sigma_{2}^{2}}{2} \mathcal{R}^{4} .
$$

This is in precise agreement with (3.12).

- In $D=8$, we get, from (2.13) and (2.42),

$$
\begin{aligned}
& \mathcal{E}_{(0,0)}^{(2)}(\varphi, L) \sim 2 \pi \log L \\
& \mathcal{E}_{(0,1)}^{(2)}(\varphi, L) \sim \frac{\pi}{3} \mathcal{E}_{(0,0)}^{(2)} \log L+\frac{2 \pi^{2}}{3}(\log L)^{2} \sim \frac{\pi}{3} \mathcal{E}_{(0,0)}^{(2)}(\varphi, L) \log L
\end{aligned}
$$

The first divergence, proportional to $\mathcal{R}^{4}$, is simply the one-loop divergence in supergravity [4]. The second divergence, proportional to $\nabla^{6} \mathcal{R}^{4}$, comes both from the oneloop divergence of the $\mathcal{R}^{4}$ form factor (3.2), and from the two-loop divergence (3.5)

\footnotetext{
${ }^{14}$ This differs by a factor 4 from the identification stated in [6, appendix B], which is necessary to match the string divergences with results in the supergravity literature for all dimensions and loop orders relevant in this paper. The matching of primitive divergences is in fact guaranteed by the fact that the tropical limit of the string integrand reproduces the supergravity integrand in Schwinger-type parametrization [42].
} 
in supergravity. The first term is guaranteed to be correctly normalized, since it contains the one-loop subdivergence of the genus-two amplitude, which is proportional to the $\mathcal{R}^{4}$ coupling. To check the normalization of the two-loop divergence, we quote again the result in $[25,(4.4)]$,

$$
\mathcal{I}_{2, \text { subtracted }}^{D=8-2 \epsilon} \sim\left(\frac{\kappa}{2}\right)^{6} \frac{1}{2(4 \pi)^{8}}\left[-\frac{1}{24 \epsilon^{2}}+\frac{1}{144 \epsilon}\right] \frac{(s t u)^{2}}{3} M_{4}^{\text {tree }} \sim-\frac{2 \pi^{2}}{3}(\log L)^{2} \sigma_{3} \mathcal{R}^{4},
$$

where we identified $1 / \epsilon^{2} \sim \frac{1}{2}(\log L)^{2}$, consistently with $\frac{L^{h \epsilon}}{\epsilon^{2}}=\frac{1}{\epsilon^{2}}+h \frac{\log L}{\epsilon}+\frac{1}{2} h^{2}(\log L)^{2}+$ .... Comparing with the last term in (3.15), we see precise agreement, up to an overall sign. This sign difference is in fact expected, since the result (3.16) refers to the subtracted amplitude, where the one-loop subdivergence has been cancelled against the triangle diagram with a one-loop counterterm. As explained in [6], the relative normalisation of the sunset and triangle diagrams is fixed by demanding that the coefficient of the $1 / \epsilon$ pole in dimensional regularisation is analytic in the Mandelstam variables, and leads to a sign flip in the coefficient of the $1 / \epsilon^{2}$ pole.

- In $D=7$, we get, from $(2.41)$,

$$
\mathcal{E}_{(1,0)}^{(3)}(\varphi, L) \sim \frac{4 \pi^{2}}{3} \log L
$$

This should come from the two-loop divergence (3.18) in supergravity. To check the numerical factor, we quote the result in [25],

$$
\mathcal{I}_{2}^{D=7-2 \epsilon} \sim\left(\frac{\kappa}{2}\right)^{6} \frac{\pi}{3} \frac{s^{2}+t^{2}+u^{2}}{2 \epsilon(4 \pi)^{7}} s t u M_{4}^{\text {tree }}=\frac{4 \pi^{2}}{3} \log L \sigma_{2} \mathcal{R}^{4},
$$

in perfect agreement with (3.17). In particular, the factor $\pi / 3$ in (3.18) is identified as the volume of the fundamental domain $\mathcal{F}$.

- In $D=6$, we get, from $(2.16),(2.41),(2.43),(2.47)$,

$$
\begin{aligned}
& \mathcal{E}_{(1,0)}^{(4)}(\varphi, L) \sim \mathcal{E}_{(0,0)}^{(4)} \log L \\
& \mathcal{E}_{(0,1)}^{(4)}(\varphi, L) \sim 5 \zeta(3) \log L \\
& \mathcal{E}_{(2,0)}^{(4)}(\varphi, L) \sim \frac{5}{3} \mathcal{E}_{(1,0)}^{(4)} \log L+\frac{7}{6} \mathcal{E}_{(0,0)}^{(4)}(\log L)^{2}
\end{aligned}
$$

The first divergence, proportional to $\nabla^{4} \mathcal{R}^{4}$, comes from the one-loop divergence of the $\mathcal{R}^{4}$ form factor (3.2) (which includes a contribution from the one-loop subdivergence of the genus-two amplitude). The divergence on the third line, proportional to $\nabla^{8} \mathcal{R}^{4}$, may be rewritten as

$$
\mathcal{E}_{(2,0)}^{(4)}(\varphi, L) \sim \frac{5}{3} \mathcal{E}_{(1,0)}^{(4)}(\varphi, L) \log L-\frac{1}{2} \mathcal{E}_{(0,0)}^{(4)}(\log L)^{2},
$$

exhibiting a contribution from the one-loop divergence of the $\nabla^{4} \mathcal{R}^{4}$ form factor (3.3), and from the two-loop divergence of the $\mathcal{R}^{4}$ form factor (3.6). Note that (3.20) 
predicts a divergence proportional to $\mathcal{E}_{(1,0)}^{(4,2)}(\varphi) \log L$, which should arise from a oneloop subdivergence in the genus three contribution to the $\nabla^{8} \mathcal{R}^{4}$ coupling (2.48). Finally, the divergence on the second line of (3.19), proportional to $\nabla^{6} \mathcal{R}^{4}$, should come from the three-loop divergence (3.21). To check the numerical coefficient, we quote the result in $[26,(5.12)]$,

$$
\mathcal{I}_{3}^{D=6-2 \epsilon} \sim \frac{5 \zeta(3)}{(4 \pi)^{9} \epsilon}\left(\frac{\kappa}{2}\right)^{8}(s t u)^{2} M_{4}^{\text {tree }}=5 \zeta(3) \log L \sigma_{3} \mathcal{R}^{4},
$$

where we identified $1 / \epsilon \sim 3 \log L$ as appropriate at 3-loop order. This is in precise agreement with the coefficient of $\log L$ in (3.19).

- In $D=5$, we get, from (2.42),

$$
\mathcal{E}_{(0,1)}^{(5)}(\varphi, L) \sim \frac{5}{3} \mathcal{E}_{(0,0)}^{(5)} \log L
$$

This comes from the two-loop divergence of the $\mathcal{R}^{4}$ form factor (3.6), which is correctly normalized since it includes a contribution from the two-loop subdivergence of the genus-three amplitude (2.47).

- In $D=4$, we get, from (2.15), (2.16), (2.42), (2.43),

$$
\begin{aligned}
& \mathcal{E}_{(0,1)}^{(6)}(\varphi, L) \sim \frac{5}{2 \pi} \mathcal{E}_{(1,0)}^{(6)} \log L \\
& \mathcal{E}_{(2,0)}^{(6)}(\varphi, L) \sim \frac{1}{4 \pi}\left(3 \mathcal{E}_{(0,1)}^{(6)}+\frac{1}{2}\left(\mathcal{E}_{(0,0)}^{(6)}\right)^{2}\right) \log L+\frac{3}{2 \pi^{2}} \mathcal{E}_{(1,0)}^{(6)}(\log L)^{2}
\end{aligned}
$$

Ignoring possible contamination from infrared effects, the first divergence, proportional to $\nabla^{6} \mathcal{R}^{4}$, should come from the one-loop divergence of the $\nabla^{4} \mathcal{R}^{4}$ form factor (3.3) (which is correctly normalized since it includes a contribution from the one-loop subdivergence of the genus-three amplitude (2.47)). The second divergence, proportional to $\nabla^{8} \mathcal{R}^{4}$, may be rewritten as

$$
\mathcal{E}_{(2,0)}^{(6)}(\varphi, L) \sim \frac{1}{8 \pi}\left(\mathcal{E}_{(0,0)}^{(6)}\right)^{2} \log L+\frac{3}{4 \pi} \mathcal{E}_{(0,1)}^{(6)}(\varphi, L) \log L-\frac{3}{8 \pi^{2}} \mathcal{E}_{(1,0)}^{(6)}(\log L)^{2},
$$

exhibiting the one-loop divergence of the $\nabla^{6} \mathcal{R}^{4}$ and $\left(\mathcal{R}^{4}\right)^{2}$ form factors (3.4), along with the two-loop divergence of the $\nabla^{4} \mathcal{R}^{4}$ form factor (3.7). Note that (3.24) predicts divergences of the form $\mathcal{E}_{(0,1)}^{(6,2)}(\log L), \mathcal{E}_{(1,0)}^{(6,1)}(\log L)^{2}$, which should arise from subdivergences of the genus-three amplitude (2.48). Similarly, the term $\mathcal{E}_{(1,0)}^{(6,2)}(\log L)^{2}$ should arise from a two-loop subdivergence of the genus-four string amplitude at order $\nabla^{8} \mathcal{R}^{4}$, which is currently out of reach.

- In $D=3$, we get, from (2.43),

$$
\mathcal{E}_{(2,0)}^{(7)}(\varphi, L) \sim \frac{2 \beta \zeta(3)^{2}}{\pi^{3}} \log L
$$

Barring possible effects from infrared divergences, this should come from the two-loop divergence of the $\nabla^{6} \mathcal{R}^{4}$ and $\left(\mathcal{R}^{4}\right)^{2}$ form factors (3.8). Unfortunately, we do not know how to derive this coefficient independently. 


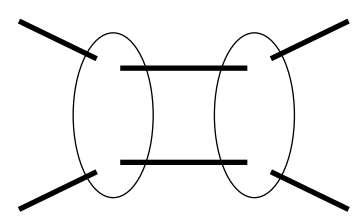

(a)

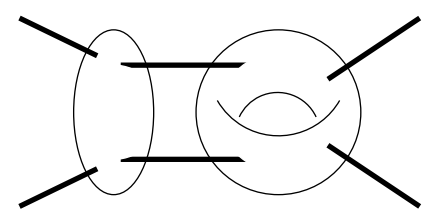

(b)

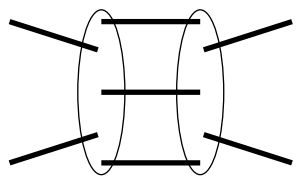

(c)

Figure 5. (a) Discontinuity of the one-loop amplitude. (b) Two-particle discontinuity of the two-loop amplitude. (c) Three-particle discontinuity.

\section{Discussion}

In this note, we studied effective interactions of the form $\mathcal{E}_{(p, q)}^{(d)}(\varphi, L) \nabla^{4 p+6 q} \mathcal{R}^{4}$ in the Wilsonian effective action $\mathcal{S}(\Lambda)$ describing interactions of massless particles at low energy in type II strings compactified on $T^{d}$. Perturbatively, these Wilsonian couplings are defined by restricting the integration over the moduli space $\mathcal{M}_{h, n}$ of Riemann surfaces to the subset where the proper time associated to any handle is bounded by $L=1 /\left(\alpha^{\prime} \Lambda^{2}\right)$. Using recently available information on the asymptotics of the integrands near boundaries of moduli space at genus one [14] and two [21, 23, 30], we computed the large $L$ behavior of the coefficients $\mathcal{E}_{(p, q)}^{(d)}(\varphi, L)$, and matched these terms against the UV divergences arising in maximal supergravity with a UV cut-off $\Lambda$, supplemented by higher-derivative stringy interactions. Since supergravity amplitudes are more easily computed in dimensional regularisation, we focused on logarithms arising in various dimensions, at various order in the low energy expansion, although one could in principle also try to match power divergences in presence of a hard cut-off.

This matching gives a strong consistency check on the structure of the asymptotic expansion of the integrands $\mathcal{B}_{(p, q)}^{(h)}$ near boundaries of moduli space, in particular on the powers of the degeneration parameter $t$ or $V$ appearing in the Laurent expansion, and it elucidates the origin of the transcendental coefficients which appear in these expansions. It follows from our analysis that the genus-one modular graph functions appearing in the expansion (2.37) at large $t$ are directly related to the genus-one integrands (2.3), while the coefficients $\zeta(3), \zeta(5), \zeta(3)^{2}$ in the expansion (2.39) in the tropical limit $V \rightarrow 0$ are directly related to the tree-level interactions (1.3). In particular, this rules out a term proportional to $\zeta(7)$, which appears in intermediate computations in [23] but cancels in the final result. Combined with U-duality, this structure also constrains the degenerations of the integrands at higher genus, even though the full integrand is yet to be computed beyond genus two. At the non-perturbative level, U-duality implies that instanton corrections must also contribute divergent terms, although the Wilsonian effective action remains to be defined beyond perturbation theory.

While the Wilsonian couplings depend on the cut-off $L$ and have the usual field redefinition ambiguities, the full on-shell scattering amplitude (1.1) is unambiguous and independent of $L$ (though it would depend on the IR cut-off in $D \leq 4$ ). In dimensions where logarithms are present, this requires a cancellation between analytic terms 
of the form $\log L \sigma_{2}^{p} \sigma_{3}^{q} \mathcal{R}^{4}$ coming from the second term in (1.1), and non-analytic contributions of the form $-\log \left(-s L \ell_{s}^{2}\right) \sigma_{2}^{p} \sigma_{3}^{q} \mathcal{R}^{4}$ from the first term, leaving a term of the form $-\log \left(-s \ell_{s}^{2}\right) \sigma_{2}^{p} \sigma_{3}^{q} \mathcal{R}^{4}$ in the amplitude. The latter are in turn computable by unitarity, since they contribute to the imaginary part of the forward amplitude. This mechanism was studied in detail at genus-one in [13], where unitarity was used to relate the discontinuity of the one-loop amplitude to the square of the four-point tree-level amplitude (diagram $(a)$ in figure 5).

The same unitarity argument can in principle be applied at genus-two, but is considerably more involved. Indeed, one has to include both the diagram (b) with two-particle intermediate states, involving the product of a tree-level and one-loop four-point amplitudes, and the diagram $(c)$ with three-particle intermediate states, involving the product of two tree-level five-point amplitudes. In fact, rather than working order by order in the genus expansion, it is more expedient to work at the non-perturbative level and express the imaginary part of the forward amplitude as a sum of squares of on-shell $N+2$-point functions, where $N$ is the number of intermediate states (for $N=2$ and $N=3$, this corresponds to the diagrams $(a)$ and $(c)$, where the oval now stands for the full on-shell $N+2$-point function).

Approximating the 4-point function in diagram $(a)$ and 5-point function in diagram (b) by tree-level supergravity vertices, the sum over intermediate states and integral over the corresponding phase space produces the discontinuity of the corresponding one-loop and two-loop supergravity diagrams, from which one can read off the analytic structure of the amplitude. At one and two loops, the latter is schematically of the form [25]

$$
s^{\frac{D-8}{2}} \log (-s) \mathcal{R}^{4}+s^{D-7} \log (-s) \nabla^{4} \mathcal{R}^{4}
$$

These terms are indeed consistent ${ }^{15}$ with the $\log L$ terms predicted by (3.1) and (3.5) in any dimension $D$. Including higher-derivative corrections of the form $\mathcal{E}_{(p, q)}^{(d)} \nabla^{4 p+6 q} \mathcal{R}^{4}$ to the onshell quartic vertices in diagram $(a)$, one obtains a series of corrections to the discontinuity, and therefore to the amplitude itself, of the form

$$
\begin{aligned}
\mathcal{E}_{(0,0)}^{(d)} s^{\frac{D-2}{2}} \log (-s) \mathcal{R}^{4}+\mathcal{E}_{(1,0)}^{(d)} s^{\frac{D+2}{2}} \log ( & -s) \mathcal{R}^{4} \\
& +\left[\mathcal{E}_{(0,1)}^{(d)}+\left(\mathcal{E}_{(0,0)}^{(d)}\right)^{2}\right] s^{\frac{D+4}{2}} \log (-s) \mathcal{R}^{4}+\ldots
\end{aligned}
$$

These terms agree with the $\log L$ terms from (3.2), (3.3), (3.4), etc, justifying the use of onshell vertices in these computations. In addition, there are also higher-derivative corrections to the on-shell five-point functions in diagram (b), of the form $\nabla^{2 n} \mathcal{R}^{5}$ with $n \geq 1$ [39, 40]. A subset of those corrections are related by supersymmetry to the $\nabla^{4 p+6 q} \mathcal{R}^{4}$ quartic couplings with $2 n=4 p+6 q-2$ [41]. For those couplings, which are essentially obtained by connecting

\footnotetext{
${ }^{15}$ In $D=8$, there is also a term proportional to $[\log (-s)]^{2} \nabla^{6} \mathcal{R}^{4}$, which is necessary to cancel $(\log L)^{2} \nabla^{6} \mathcal{R}^{4}$ from the Wilsonian coupling. In this sketchy discussion we ignore such effects, related to one-loop subdivergences of the two-loop amplitude.
} 
a quartic and cubic vertex using a propagator, we expect contributions of the form

$$
\begin{aligned}
\mathcal{E}_{(0,0)}^{(d)} s^{D-4} \log (-s) \nabla^{4} \mathcal{R}^{4}+\mathcal{E}_{(1,0)}^{(d)} s^{D-2} & \log (-s) \nabla^{4} \mathcal{R}^{4} \\
& +\left[\mathcal{E}_{(0,1)}^{(d)}+\left(\mathcal{E}_{(0,0)}^{(d)}\right)^{2}\right] s^{D-1} \log (-s) \nabla^{4} \mathcal{R}^{4}+\ldots
\end{aligned}
$$

in agreement with the results (3.6), (3.7), (3.8) of the naive dimensional analysis. However, there are also corrections to the five-point function which are not related to four-point vertices by supersymmetry [41]. The leading such interaction, of order $\nabla^{6} \mathcal{R}^{5}$, will produce a term of the form $s^{D} \log (-s) \nabla^{4} \mathcal{R}^{4}$, which predicts logarithmic divergences at order $\nabla^{12} \mathcal{R}^{4}$ in $D=4$, far beyond the order considered in this paper.

As pointed out in [6], upon converting the non-analytic term $-\log \left(-s \ell_{s}^{2}\right) \sigma_{2}^{p} \sigma_{3}^{q} \mathcal{R}^{4}$ in the amplitude from string frame to Einstein frame, using $\ell_{s}=\ell_{D} g_{D}^{2 /(2-D)}$, a term of the form $\alpha \log L$ in the Wilsonian coupling $\mathcal{E}_{(p, q)}^{(d)}(\varphi, L)$ implies a non-analytic term $\frac{4 \alpha}{D-2} \log g_{D}$ in the weak coupling expansion of the U-duality invariant coefficient $\mathcal{E}_{(p, q)}^{(d)}(\varphi)$. Similarly, by the same logic as in the discussion of the $\nabla^{6} \mathcal{R}^{4}$ interaction in $D=8$ [6], a term of the form $\tilde{\alpha}(\log L)^{2}$ in $\mathcal{E}_{(p, q)}^{(d)}(\varphi, L)$ can be seen to imply a non-analytic term ${ }^{16}-\frac{1}{2} \tilde{\alpha}\left(\frac{4}{D-2} \log g_{D}\right)^{2}$. It follows from the results in this note at order $\nabla^{8} \mathcal{R}^{4}$ that the U-duality invariant coefficient $\mathcal{E}_{(2,0)}^{(d)}$ must contain the terms

$$
\begin{aligned}
\mathcal{E}_{(2,0)}^{(d), \text { non-an. }}= & {\left[-\frac{1}{3} \mathcal{E}_{(0,0)}^{(4)} \delta_{d, 4}-\frac{81}{4 \pi^{2}} \mathcal{E}_{(1,0)}^{(6)} \delta_{d, 6}\right]\left(\log g_{D}\right)^{2} } \\
+ & {\left[\frac{2 \pi^{2}}{45} \mathcal{E}_{(0,0)}^{(0)} \delta_{d, 0}-\frac{416 \pi^{4}}{3969} \delta_{d, 1}+\frac{5}{3}\left(\mathcal{E}_{(1,0)}^{(4)}+\gamma \mathcal{E}_{(0,0)}^{(4)}\right) \delta_{d, 4}\right.} \\
& \left.+\frac{1}{2 \pi}\left(3 \mathcal{E}_{(0,1)}^{(6)}+\eta \mathcal{E}_{(1,0)}^{(6)}+\frac{1}{2}\left(\mathcal{E}_{(0,0)}^{(6)}\right)^{2}\right) \delta_{d, 6}+\frac{8 \beta \zeta(3)^{2}}{\pi^{3}} \delta_{d, 3}+\ldots\right] \log g_{D}
\end{aligned}
$$

generalizing similar results at order $\mathcal{R}^{4}, \nabla^{4} \mathcal{R}^{4}$ and $\nabla^{6} \mathcal{R}^{4}[6,9,10]$. In (4.4), the dots indicate contributions which could in principle arise at genus 3 or higher, and the coefficients $\gamma, \eta$ cannot be determined from this sketchy argument.

Thus, we see that the structure of the logarithms in the coefficients of the Wilsonian effective interactions is closely reflected by the analyticity properties of the scattering amplitude in a low energy expansion, and by the non-analytic terms in the weak coupling expansion of the U-duality invariant coefficients. A more detailed discussion of these connections, including effects of subdivergences at genus two and beyond, is left for future work.

\section{Acknowledgments}

I wish to thank Eric d'Hoker and Michael B. Green for collaboration on [23] where the problem addressed in this note was raised, and for collaborating at an initial stage on this follow-up project. I am also grateful to Ashoke Sen, Guillaume Bossard, Henrik Johansson and Roji Pius for helpful discussions. My research of is supported in part by French

\footnotetext{
${ }^{16}$ In particular, the divergent terms in (3.15) imply $\mathcal{E}_{(0,1)}^{(2), \text { non-an. }}=-\frac{4 \pi^{2}}{27}\left(\log g_{8}\right)^{2}+\frac{2 \pi}{9}\left(\mathcal{E}_{(0,0)}^{(2)}+\right.$ cte $) \log g_{8}$, in agreement with $[9,(2.19)]$.
} 
state funds managed by ANR in the context of the LABEX ILP (ANR-11-IDEX-0004-02, ANR-10-LABX-63).

\section{A Constraints on $\nabla^{10} \mathcal{R}^{4}$ interactions}

In this section, we briefly discuss the implications of UV divergences in supergravity for the string integrands computing $\nabla^{10} \mathcal{R}^{4}$ interactions in the low energy effective action. At genus one, the integrand was computed in $[14,(7.28)]$,

$$
\mathcal{B}_{(1,1)}^{(1)}=\frac{5}{6 !}\left(336 C_{3,1,1}+240 E_{2} E_{3}+48 \zeta(3) E_{2}-\frac{1632}{5} E_{5}+\frac{144}{5} \zeta(5)\right)
$$

where $C_{3,1,1}$ is one of the modular graph functions defined in [14]. Using the asymptotics derived in $[14,(6.2)]$, we have

$\mathcal{B}_{(1,1)}^{(1)}=\frac{4 \pi^{5} \tau_{2}^{5}}{66825}+\frac{\pi^{2} \tau_{2}^{2} \zeta(3)}{63}+\frac{29 \zeta(5)}{135}+\frac{\zeta(3)^{2}}{3 \pi \tau_{2}}+\frac{49 \zeta(7)}{48 \pi^{2} \tau_{2}^{2}}+\frac{\zeta(3) \zeta(5)}{12 \pi^{3} \tau_{2}^{3}}+\frac{21 \zeta(9)}{64 \pi^{4} \tau_{2}^{4}}+\mathcal{O}\left(e^{-2 \pi \tau_{2}}\right)$

The resulting divergent terms in the truncated modular integral are given by ${ }^{17}$

$$
\begin{aligned}
\mathcal{E}_{(1,1)}^{(d, 1)}(\varphi, L)= & \frac{8 \pi^{6}}{66825} \frac{L^{\frac{d+8}{2}}}{\frac{d+8}{2}}+\frac{2 \pi^{3} \zeta(3)}{63} \frac{L^{\frac{d+2}{2}}}{\frac{d+2}{2}}+\frac{58 \pi \zeta(5)}{135} \frac{L^{\frac{d-2}{2}}}{\frac{d-2}{2}}+\frac{2 \zeta(3)^{2}}{3} \frac{L^{\frac{d-4}{2}}}{\frac{d-4}{2}} \\
& +\frac{49 \zeta(7)}{24 \pi} \frac{L^{\frac{d-6}{2}}}{\frac{d-6}{2}}+\frac{\zeta(3) \zeta(5)}{6 \pi^{2}} \frac{L^{\frac{d-8}{2}}}{\frac{d-8}{2}}+\frac{21 \zeta(9)}{32 \pi^{3}} \frac{L^{\frac{d-10}{2}}}{\frac{d-10}{2}}+\mathcal{E}_{(1,1)}^{(d, 1)}(\varphi)
\end{aligned}
$$

At genus two, the integrand $\mathcal{B}_{(1,1)}^{(2)}$ is a modular graph function of weight 3 , given by an integral of a sum of products of three Arakelov-Green functions over the location of the four vertices $[19,(4.2)]$. The complete asymptotics of this modular graph function has not been fully analyzed yet, but its leading tropical limit is known from the supergravity computation in $[24,(\mathrm{~A} .22)] \cdot{ }^{18}$ Expressing the result in terms of the modular local Laurent polynomials defined in appendix B, we get

$$
\begin{aligned}
\mathcal{B}_{(1,1)}^{(2)}= & \frac{4 \pi^{3}}{405 V^{3}}\left[-\frac{35}{66} A_{0,1}+\frac{679}{429} A_{0,3}-\frac{2238}{143} A_{1,0}+\frac{147}{13} A_{1,2}+\frac{266}{17} A_{2,1}+\frac{145}{14} A_{3,0}\right] \\
& +\zeta(3) \tilde{\mathcal{B}}_{(1,1)}^{(0)}+\frac{\zeta(5)}{\pi^{2}} V^{2} \tilde{\mathcal{B}}_{(1,1)}^{(2)}+\frac{\zeta(3)^{2}}{\pi^{3}} V^{3} \tilde{\mathcal{B}}_{(1,1)}^{(3)}+\frac{\zeta(7)}{\pi^{4}} V^{4} \tilde{\mathcal{B}}_{(1,1)}^{(4)} \\
& +\frac{\zeta(3) \zeta(5)}{\pi^{5}} V^{5} \tilde{\mathcal{B}}_{(1,1)}^{(5)}+\frac{V^{6}}{\pi^{6}}\left[\zeta(9) \tilde{\mathcal{B}}_{(1,1)}^{(6)}+\zeta(3)^{3} \tilde{\mathcal{B}}_{(1,1)}^{\left(6^{\prime}\right)}\right]+\mathcal{O}\left(e^{-1 / \sqrt{V}}\right) .
\end{aligned}
$$

The coefficients of the subleading terms on the second line are not known, but they are expected to be linear combinations of the functions $A_{i, j}$. Rewriting this result in the

\footnotetext{
${ }^{17}$ For $d=0$, this reduces to $[13,(3.22)]$, up to normalisation. The finite term $\mathcal{E}_{(1,1)}^{(0,1)}(\varphi)$ in $D=10 / d=0$ is computed in [14, section 7.4].

${ }^{18}$ The precise normalisation is given in $[38,(4.8)]$. Note that these authors integrate over a six-fold cover of the domain $\mathcal{F}$, with no ordering on $L_{1}, L_{2}, L_{3}$, with measure $\mathrm{d}^{3} \Omega=\mathrm{d} L_{1} \mathrm{~d} L_{2} \mathrm{~d} L_{3}=V^{2} \mathrm{~d} V \mathrm{~d} \mu(S)$.
} 
variables $\tau_{2}=1 /\left(V S_{2}\right), t=S_{2} / V$ and reexpanding as $t \rightarrow \infty$, we find that the leading terms can be expressed in terms of genus-one modular graph functions as follows,

$$
\begin{aligned}
\mathcal{B}_{(1,1)}^{(2)}(\Omega)= & \frac{2 \pi^{3} t^{3}}{63}+\frac{4 \pi^{2} t^{2}}{27} g_{1}(\tau, v)+\frac{\pi t}{270}\left(157 E_{2}(\tau)+75 g_{1}(\tau, v)^{2}+68 g_{2}(\tau, v)\right) \\
& +\sum_{n=0}^{3} \hat{\mathcal{B}}_{(1,1)}^{(n)}(\tau, v) t^{-n}+\mathcal{O}\left(e^{-2 \pi t}\right)
\end{aligned}
$$

Here, the coefficients $\hat{\mathcal{B}}_{(1,1)}^{(n)}(\tau, v)$ are modular graph functions of weight $n+3$. Extracting the zero-th Fourier-Jacobi coefficient (see footnote 8 ), we get

$$
\int_{\left[-\frac{1}{2}, \frac{1}{2}\right]^{3}} \mathrm{~d} u_{1} \mathrm{~d} u_{2} \mathrm{~d} \sigma_{1} \mathcal{B}_{(1,1)}^{(2)}(\Omega)=\frac{2 \pi^{3}}{63} t^{3}+\frac{116 \pi t}{135} E_{2}+\ldots
$$

The divergent terms in the truncated genus two integral are therefore

$$
\begin{aligned}
\mathcal{E}_{(1,1)}^{(d, 2)}(\varphi, L)= & \frac{8 \pi^{4}}{405} \frac{L^{d}}{d}\left[-\frac{35}{66} F_{01}+\frac{679}{429} F_{03}-\frac{2238}{143} F_{10}+\frac{147}{13} F_{12}+\frac{266}{17} F_{21}+\frac{145}{14} F_{30}\right](d) \\
& +2 \pi \zeta(3) \frac{\Lambda^{d-3}}{d-3} \int_{\mathcal{F}} \mathrm{d} \mu \tilde{\mathcal{B}}_{(1,1)}^{(0)}+\frac{2 \zeta(5)}{\pi} \frac{\Lambda^{d-5}}{d-5} \int_{\mathcal{F}} \mathrm{d} \mu \tilde{\mathcal{B}}_{(1,1)}^{(2)} \\
& +\frac{2 \zeta(3)^{2}}{\pi^{2}} \frac{\Lambda^{d-6}}{d-6} \int_{\mathcal{F}} \mathrm{d} \mu \tilde{\mathcal{B}}_{(1,1)}^{(3)}+\frac{2 \zeta(7)}{\pi^{3}} \frac{\Lambda^{d-7}}{d-7} \int_{\mathcal{F}} \mathrm{d} \mu \tilde{\mathcal{B}}_{(1,1)}^{(4)}+\ldots \\
& +\frac{\pi^{3}}{63} \frac{L^{\frac{d+2}{2}}}{\frac{d+2}{2}} \mathcal{E}_{(0,0)}^{(d, 1)}(\varphi)+\frac{58 \pi}{135} \frac{L^{\frac{d-2}{2}}}{\frac{d-2}{2}} \mathcal{E}_{(1,0)}^{(d, 1)}(\varphi) \\
& +\frac{\pi}{2} \frac{L^{\frac{d-4}{2}}}{\frac{d-4}{2}} \int_{\mathcal{F}_{1}} \mathrm{~d} \mu_{1} \hat{\mathcal{B}}_{(1,1)}^{(0)} \Gamma_{d, d, 1}(\varphi)+\frac{\pi}{2} \frac{L^{\frac{d-6}{2}}}{\frac{d-6}{2}} \int_{\mathcal{F}_{1}} \mathrm{~d} \mu_{1} \hat{\mathcal{B}}_{(1,1)}^{(1)} \Gamma_{d, d, 1}(\varphi)+\ldots
\end{aligned}
$$

where the dots denote additional $L$-dependent terms on which we have little control.

Using (B.17), we find that the first term in (A.7) leads to a logarithmic divergence in $D=10$,

$$
\mathcal{E}_{(1,1)}^{(0,2)}(\varphi, L) \sim-\frac{26 \pi^{4}}{3645} \log L
$$

which is in precise agreement with the two-loop divergence computed in [25],

$$
\mathcal{I}_{2}^{D=10-2 \epsilon} \sim-\left(\frac{\kappa}{2}\right)^{6} \frac{13}{25920} \frac{\left(s^{2}+t^{2}+u^{2}\right) s t u}{12 \epsilon(4 \pi)^{10}} \text { stu } M_{4}^{\text {tree }}=-\frac{26 \pi^{4}}{3645} \log L \sigma_{2} \sigma_{3} \mathcal{R}^{4} .
$$

In particular, the pole at $d=0$ is of first order, despite the fact that the various contributions in the first line of (A.7) are separately of order $1 / d^{2}$ (see (B.17)). The structure of the remaining terms on the second and third line of (A.9) is consistent with the form factor divergences arising at two-loop in (3.6), (3.7), (3.8), and similarly for the $\nabla^{8} \mathcal{R}^{4}$ form factor divergence proportional to $\zeta(7)$. The last term on the fourth line, corresponding to the one-loop divergence of the $\nabla^{4} \mathcal{R}^{4}$ form factor (3.3) in $D=8$, nicely combines with the term proportional to $\zeta(5)$ in (A.3) so as to produce

$$
\mathcal{E}_{(1,1)}^{(2)}(\varphi, L) \sim \frac{58 \pi}{135} \mathcal{E}_{(1,0)}^{(2)}(\varphi) \log L,
$$


in turn predicting a one-loop subdivergence in the genus-three integrand at order $\nabla^{10} \mathcal{R}^{4}$. Requiring that the coefficients of the logarithmic divergence in $D=4$ recombines into the U-duality invariant coupling $\mathcal{E}_{(2,0)}^{(6)}$, we predict ${ }^{19}$

$$
\int_{\left[-\frac{1}{2}, \frac{1}{2}\right]^{3}} \mathrm{~d} u_{1} \mathrm{~d} u_{2} \mathrm{~d} \sigma_{1} \hat{\mathcal{B}}_{(1,1)}^{(1)}=\frac{49}{12 \pi^{2}} \mathcal{B}_{(2,0)}^{(1)}=\frac{49}{144 \pi^{2}}\left(D_{4}+9 E_{2}^{2}+6 E_{4}\right)
$$

Similarly, requiring that the coefficients of the logarithmic divergence in $D=6$ recombines into a linear combination of the U-duality invariant couplings $\mathcal{E}_{(0,1)}^{(4)}$ and $\left[\mathcal{E}_{(0,0)}^{(4)}\right]^{2}$ with rational coefficients, we see that the constant term of $\hat{\mathcal{B}}_{(1,1)}^{(0)}$ must be a linear combination of $E_{3}$ and $\zeta(3)$.

\section{B Some properties of modular local polynomials}

In the tropical limit $V \rightarrow 0$, the genus-two integrands have a finite Laurent series expansion

$$
\mathcal{B}_{(p, q)}^{(2)}(\Omega)=\sum_{n=-(2 p+3 q-2)}^{4 p+6 q-4} V^{n} \tilde{\mathcal{B}}_{(p, q)}^{(n)}(S)+\mathcal{O}\left(e^{-1 / \sqrt{V}}\right)
$$

where $\tilde{\mathcal{B}}_{(p, q)}^{(n)}(S)$ are modular local Laurent polynomials, a class of non-holomorphic modular functions first encountered in the study of two-loop supergravity amplitudes [24] and further developed in the mathematics literature $[43,44]$. These functions are invariant under the action of $\mathrm{GL}(2, \mathbb{Z})$ by fractional linear transformations,

$$
S \mapsto \begin{cases}\frac{a S+b}{c S+d} & a d-b c=1 \\ \frac{a \bar{S}+b}{c \bar{S}+d} & a d-b c=-1\end{cases}
$$

but they are not everywhere smooth, hence not a modular function in the usual sense. Their singular locus is given by the union of real-codimension one loci

$$
a|S|^{2}+b S_{1}+c=0, \quad b^{2}-4 a c=1,
$$

corresponding to the tropical limit of the separating degeneration divisor $v=0$ and its images under $\operatorname{Sp}(4, \mathbb{Z})$. Inside the standard fundamental domain of $\operatorname{GL}(2, \mathbb{Z})$,

$$
\mathcal{F}=\left\{S=S_{1}+\left.\mathrm{i} S_{2} \quad\left|\quad 0<S_{1}<\frac{1}{2}, \quad\right| S\right|^{2}>1\right\}=\mathcal{F}_{1} / \mathbb{Z}_{2},
$$

they are Laurent polynomial in $S_{2}$, with coefficients which are polynomial in $S_{1}$, invariant under $S_{1} \rightarrow 1-S_{1}$. A basis of such local modular functions is given by [23, 45]

$$
A_{i, j}=D_{-2 n}^{(n)}\left(u^{i} v^{j}\right)
$$

where

$$
u=S^{2}(S-1)^{2}, \quad v=S^{2}-S+1, \quad n=3 i+j .
$$

\footnotetext{
${ }^{19}$ Here we ignore a possible contribution of the diagram $(c)$ in figure 3 , involving a $\nabla^{6} \mathcal{R}^{5}$ quintic coupling unrelated to $\nabla^{8} \mathcal{R}^{4}$ by non-linear supersymmetry, of the type considered in [41].
} 
The operator $D_{-2 n}^{(n)}$ is the $n$-th iteration of the Maass raising operator acting on modular forms of weight $-2 n$,

$$
D_{-2 n}^{(n)}=\frac{(-2 i)^{n} n !}{(2 n) !} \sum_{m=0}^{n}\left(\begin{array}{c}
n \\
m
\end{array}\right) \frac{(-n-m)_{m}}{(S-\bar{S})^{m}} \frac{\partial^{n-m}}{\partial S^{n-m}}
$$

where $(k)_{m}=k(k+1) \ldots(k+m-1)$ is the Pochhammer symbol. In the standard fundamental domain, the modular function $A_{i, j}$ takes the form

$$
A_{i, j}(S)=\sum_{k=0}^{2 i+j} A_{i, j}^{(k)}\left(S_{1}\right) S_{2}^{i+j-2 k}
$$

where $A_{i, j}^{(k)}$ is a polynomial of degree $2 k$ in $S_{1}$, or rather a polynomial of degree $k$ in $T_{1}=S_{1}\left(1-S_{1}\right)$. Away from the singular loci (B.3), $A_{i, j}$ is an eigenmode of the Laplace operator $\Delta_{S}=S_{2}^{2}\left(\partial_{S_{1}}^{2}+\partial_{S_{2}}^{2}\right)$ on the upper half-plane,

$$
\left(\Delta_{S}-n(n+1)\right) A_{i, j}=0,
$$

where $n=3 i+j$. On the locus $S_{1}=0$, this equation acquires a source term proportional to $\delta\left(S_{1}\right)$ [13]. In order of increasing $n$, the first few $A_{i, j}$ 's are given explicitly in the standard fundamental domain by $A_{0,0}=1$ and

$$
\begin{aligned}
A_{0,1}= & S_{2}+\frac{1-T_{1}}{S_{2}} \\
A_{02}= & S_{2}^{2}+\left(1-2 T_{1}\right)+\frac{\left(T_{1}-1\right)^{2}}{S_{2}^{2}} \\
A_{1,0}= & \frac{S_{2}}{5}+\frac{1-6 T_{1}}{5 S_{2}}+\frac{T_{1}^{2}}{S_{2}^{3}} \\
A_{0,3}= & S_{2}^{3}+\left(\frac{6}{5}-3 T_{1}\right) S_{2}+\frac{3\left(5 T_{1}^{2}-7 T_{1}+2\right)}{5 S_{2}}-\frac{\left(T_{1}-1\right)^{3}}{S_{2}^{3}} \\
A_{1,1}= & \frac{S_{2}^{2}}{7}+\left(\frac{12}{35}-\frac{9 T_{1}}{7}\right)+\frac{3 T_{1}\left(5 T_{1}-3\right)+1}{7 S_{2}^{2}}-\frac{\left(T_{1}-1\right) T_{1}^{2}}{S_{2}^{4}} \\
A_{2,0}= & \frac{S_{2}^{2}}{33}+\left(\frac{10}{77}-\frac{20 T_{1}}{33}\right)+\frac{10 T_{1}\left(7 T_{1}-2\right)+1}{33 S_{2}^{2}}+\frac{2\left(3-14 T_{1}\right) T_{1}^{2}}{11 S_{2}^{4}}+\frac{T_{1}^{4}}{S_{2}^{6}} \\
A_{1,2}= & \frac{S_{2}^{3}}{9}+\left(\frac{8}{21}-\frac{4 T_{1}}{3}\right) S_{2}+\frac{2\left(35 T_{1}^{2}-25 T_{1}+4\right)}{21 S_{2}}+\frac{-28 T_{1}^{3}+36 T_{1}^{2}-12 T_{1}+1}{9 S_{2}^{3}} \\
& +\frac{\left(T_{1}-1\right)^{2} T_{1}^{2}}{S_{2}^{5}} \\
A_{3,0}= & \frac{S_{2}^{3}}{221}-\frac{21\left(22 T_{1}-5\right) S_{2}}{2431}+\frac{105\left(33 T_{1}^{2}-12 T_{1}+1\right)}{2431 S_{2}}+\frac{-924 T_{1}^{3}+378 T_{1}^{2}-42 T_{1}+1}{221 S_{2}^{3}} \\
& +\frac{3 T_{1}^{2}\left(33 T_{1}^{2}-12 T_{1}+1\right)}{17 S_{2}^{5}}-\frac{3\left(T_{1}^{4}\left(22 T_{1}-5\right)\right)}{17 S_{2}^{7}}+\frac{T_{1}^{6}}{S_{2}^{9}}
\end{aligned}
$$

It will be useful to note that except for $A_{00}$, all $A_{i j}$ vanish on the line $S_{1}=1 / 2, S_{2}>\sqrt{3} / 2$. On the line $S_{1}=0, S_{2}>1$, they are continuous but not differentiable, with the left/right 
derivative $P_{i, j}(S) \equiv \pm\left.\partial_{S_{1}} A_{i j}\right|_{S_{1}=0^{ \pm}}$given by

$$
\begin{aligned}
P_{0,1} & =-\frac{1}{S_{2}}, & P_{0,2} & =-2\left(1+\frac{1}{S_{2}^{2}}\right), \\
P_{1,1} & =-\frac{9}{7}\left(1+\frac{1}{S_{2}^{2}}\right), & P_{2,0} & =-\frac{20}{33}\left(1+\frac{1}{S_{2}^{2}}\right) \\
P_{1,2} & =-\frac{4 S_{2}}{3}-\frac{50}{21 S_{2}}-\frac{4}{3 S_{2}^{3}}, & P_{2,1} & =-\frac{75 S_{2}}{143}-\frac{490}{429 S_{2}}-\frac{75}{143 S_{2}^{3}}, \\
P_{0,3} & =-3 S_{2}-\frac{21}{5 S_{2}}-\frac{3}{S_{2}^{3}}, & P_{3,0} & =-\frac{42 S_{2}}{221}-\frac{1260}{2431 S_{2}}-\frac{42}{221 S_{2}^{3}} .
\end{aligned}
$$

It will also be useful to record the zero-mode with respect to $S_{1}$ for $S_{2}>1$,

$$
\begin{aligned}
& \int_{0}^{1 / 2} \mathrm{~d} S_{1} A_{0,1}=\frac{S_{2}}{2}+\frac{5}{12 S_{2}} \\
& \int_{0}^{1 / 2} \mathrm{~d} S_{1} A_{0,2}=\frac{S_{2}^{2}}{2}+\frac{1}{3}+\frac{7}{20 S_{2}^{2}} \\
& \int_{0}^{1 / 2} \mathrm{~d} S_{1} A_{1,0}=\frac{S_{2}}{10}+\frac{1}{60 S_{2}^{3}} \\
& \int_{0}^{1 / 2} \mathrm{~d} S_{1} A_{0,3}=\frac{S_{2}^{3}}{2}+\frac{7 S_{2}}{20}+\frac{3}{10 S_{2}}+\frac{83}{280 S_{2}^{3}} \\
& \int_{0}^{1 / 2} \mathrm{~d} S_{1} A_{1,1}=\frac{S_{2}^{2}}{14}+\frac{9}{140}+\frac{11}{840 S_{2}^{4}} \\
& \int_{0}^{1 / 2} \mathrm{~d} S_{1} A_{2,0}=\frac{S_{2}^{2}}{66}+\frac{10}{693}+\frac{11}{1260 S_{2}^{6}} \\
& \int_{0}^{1 / 2} \mathrm{~d} S_{1} A_{1,2}=\frac{S_{2}^{3}}{18}+\frac{5 S_{2}}{63}+\frac{1}{21 S_{2}}+\frac{13}{1260 S_{2}^{5}} \\
& \int_{0}^{1 / 2} \mathrm{~d} S_{1} A_{2,1}=\frac{3 S_{2}^{3}}{286}+\frac{35 S_{2}}{1716}+\frac{25}{2574 S_{2}}+\frac{17}{27720 S_{2}^{7}} \\
& \int_{0}^{1 / 2} \mathrm{~d} S_{1} A_{3,0}=\frac{S_{2}^{3}}{442}+\frac{14 S_{2}}{2431}+\frac{21}{9724 S_{2}}+\frac{1}{24024 S_{2}^{9}}
\end{aligned}
$$

Note that several of the coefficients $A_{i, j}^{(k)}\left(S_{1}\right)$ with $0<k<2 i+j$ integrate to zero.

In order to define the renormalized integral in section 2 , we need to compute integrals of the form

$$
F_{i j}(s)=\int_{\mathcal{F}} \mathrm{d} \mu S_{2}^{-s} A_{i j}(S), \quad \mathrm{d} \mu=2 \frac{\mathrm{d} S_{1} \mathrm{~d} S_{2}}{S_{2}^{2}} .
$$

Inserting the expansion (B.8), we see that the integral converges for $\operatorname{Re}(s)>i+j-1$, and that simple poles occur at $s=i+j-2 k-1$ for $k=0, \ldots 2 i+j$, with residue proportional to $\int_{0}^{1 / 2} \mathrm{~d} S_{1} A_{i, j}^{(k)}\left(S_{1}\right)$. Using (B.12) we deduce the pole structure for low values of $(i, j)$ :

- $F_{0,0}$ has simple poles at $s=-1$, with residue 1 ;

- $F_{0,1}$ has simple poles at $s \in\{0,-2\}$, with residues $\left\{1, \frac{5}{6}\right\}$, respectively; 
- $F_{0,2}$ has simple poles at $s \in\{1,-1,-3\}$, with residues $\left\{1, \frac{2}{3}, \frac{7}{10}\right\}$;

- $F_{1,0}$ has simple poles at $s \in\{0,-4\}$, with residues $\left\{\frac{1}{5}, \frac{1}{30}\right\}$;

- $F_{0,3}$ has simple poles at $s \in\{2,0,-2,-4\}$, with residue $\left\{1, \frac{7}{10}, \frac{3}{5}, \frac{83}{140}\right\}$;

- $F_{1,1}$ has simple poles at $s \in\{1,-1,-5\}$, with residues $\left\{\frac{1}{7}, \frac{9}{70}, \frac{11}{420}\right\}$;

- $F_{2,0}$ has simple poles at $s \in\{1,-1,-7\}$, with residues $\left\{\frac{1}{33}, \frac{20}{693}, \frac{1}{630}\right\}$;

- $F_{1,2}$ has simple poles at $s \in\{2,0,-2,-6\}$, with residues $\left\{\frac{1}{9}, \frac{10}{63}, \frac{2}{21}, \frac{5}{63}\right\}$;

- $F_{2,1}$ has simple poles at $s \in\{2,0,-2,-8\}$, with residues $\left\{\frac{3}{143}, \frac{35}{858}, \frac{25}{1287}, \frac{17}{13860}\right\}$;

- $F_{3,0}$ has simple poles at $s \in\{2,0,-2,-10\}$, with residues $\left\{\frac{1}{221}, \frac{28}{2431}, \frac{21}{4862}, \frac{1}{12012}\right\}$;

The full integral (2.44) can be evaluated by integration by parts, using the fact (B.9) that $A_{i, j}$ is an eigenmode of $\Delta_{S}$ away from $S_{1}=0$. Following the same steps as in [22, (2.45)], we get, for $n=3 i+j>0$,

$$
\begin{aligned}
& {\left[1-\frac{s(s+1))}{n(n+1)}\right] \int_{\mathcal{F}} \mathrm{d} \mu S_{2}^{-s} A_{i, j}(S)=\frac{1}{n(n+1)} \int_{\partial \mathcal{F}}\left(S_{2}^{-s} \star \mathrm{d} A_{i, j}-A_{i, j} \star \mathrm{d} S_{2}^{-s}\right)} \\
& =-\frac{2}{n(n+1)} \int_{1}^{\infty} P_{i j}\left(S_{2}\right) S_{2}^{-s} \mathrm{~d} S_{2}-\frac{2 s}{n(n+1)} \int_{0}^{1 / 2} \mathrm{~d} S_{1}\left(1-S_{1}^{2}\right)^{\frac{-s-1}{2}} A_{i j}\left(S_{1}, \sqrt{1-S_{1}^{2}}\right)
\end{aligned}
$$

where the two terms on the last line correspond to the boundary contribution at $S_{1}=0$ and at $|S|^{2}=1$ (there is no contribution from the boundary at $S_{1}=1 / 2$ since both $A_{i, j}$ and the normal derivative $\star \mathrm{d} A_{i, j}$ vanish there). The first integral is trivially evaluated, while the second integral is a linear combination of integrals of the form $[22,(2.37)]$

$$
c_{n}(\gamma):=\int_{0}^{1 / 2} \mathrm{~d} x x^{2 n}\left(1-x^{2}\right)^{-\gamma}=\frac{4^{-n-1}}{n+\frac{1}{2}}{ }_{1} F_{2}\left(\gamma, n+\frac{1}{2} ; n+\frac{3}{2} ; \frac{1}{4}\right) .
$$

Poles in $F_{i, j}(s)$ a priori occur when $s=n, s=-n-1$ or when $\int_{1}^{\infty} P_{i j}\left(S_{2}\right) S_{2}^{-s} \mathrm{~d} S_{2}$ has a pole, since the last term in (B.14) is smooth. In order for the apparent pole at $s=n$ to cancel, the r.h.s. of (B.14) must vanish at that value. Using this we find

$$
F_{0,0}(s)=\frac{\pi}{3}+\mathcal{O}(s), \quad F_{0,2}, F_{1,1}, F_{2,0}=\mathcal{O}(s),
$$

while

$$
\begin{aligned}
F_{0,1}(s) & =\frac{1}{s}+\frac{1}{2}+\mathcal{O}(s), & F_{1,0}(s) & =\frac{1}{5 s}+\frac{1}{60}+\mathcal{O}(s) \\
F_{1,2}(s) & =\frac{10}{63 s}+\frac{1}{189}+\mathcal{O}(s), & F_{2,1} & =\frac{35}{858 s}+\frac{5}{6864}+\mathcal{O}(s), \\
F_{0,3}(s) & =\frac{7}{10 s}+\frac{7}{120}+\mathcal{O}(s), & F_{3,0}(s) & =\frac{28}{2431 s}+\frac{14}{109395}+\mathcal{O}(s) .
\end{aligned}
$$

The finite terms in these expansions have been used in obtaining (A.8). 
Open Access. This article is distributed under the terms of the Creative Commons Attribution License (CC-BY 4.0), which permits any use, distribution and reproduction in any medium, provided the original author(s) and source are credited.

\section{References}

[1] E. D'Hoker and D.H. Phong, The Geometry of String Perturbation Theory, Rev. Mod. Phys. 60 (1988) 917 [INSPIRE].

[2] E. Witten, Superstring Perturbation Theory Revisited, arXiv:1209.5461 [INSPIRE].

[3] R. Donagi and E. Witten, Supermoduli Space Is Not Projected, Proc. Symp. Pure Math. 90 (2015) 19 [arXiv: 1304.7798] [INSPIRE].

[4] M.B. Green, J.H. Schwarz and L. Brink, $N=4$ Yang-Mills and $N=8$ Supergravity as Limits of String Theories, Nucl. Phys. B 198 (1982) 474 [inSPIRE].

[5] A. Sen, Wilsonian Effective Action of Superstring Theory, JHEP 01 (2017) 108 [arXiv: 1609.00459] [INSPIRE].

[6] M.B. Green, J.G. Russo and P. Vanhove, String theory dualities and supergravity divergences, JHEP 06 (2010) 075 [arXiv: 1002.3805] [INSPIRE].

[7] M.B. Green and P. Vanhove, The low-energy expansion of the one loop type-II superstring amplitude, Phys. Rev. D 61 (2000) 104011 [hep-th/9910056] [INSPIRE].

[8] C.M. Hull and P.K. Townsend, Unity of superstring dualities, Nucl. Phys. B 438 (1995) 109 [hep-th/9410167] [INSPIRE].

[9] B. Pioline, $D^{6} R^{4}$ amplitudes in various dimensions, JHEP 04 (2015) 057 [arXiv: 1502.03377] [INSPIRE].

[10] A. Basu, Non-analytic terms from nested divergences in maximal supergravity, Class. Quant. Grav. 33 (2016) 145007 [arXiv: 1604.02667] [INSPIRE].

[11] D.J. Gross and E. Witten, Superstring Modifications of Einstein's Equations, Nucl. Phys. B 277 (1986) 1 [INSPIRE].

[12] M.B. Green and J.H. Schwarz, Supersymmetrical String Theories, Phys. Lett. 109B (1982) 444 [INSPIRE].

[13] M.B. Green, J.G. Russo and P. Vanhove, Low energy expansion of the four-particle genus-one amplitude in type-II superstring theory, JHEP 02 (2008) 020 [arXiv:0801.0322] [INSPIRE].

[14] E. D'Hoker, M.B. Green and P. Vanhove, On the modular structure of the genus-one Type II superstring low energy expansion, JHEP 08 (2015) 041 [arXiv: 1502.06698] [INSPIRE].

[15] E. D'Hoker, M.B. Green, Ö. Gürdogan and P. Vanhove, Modular Graph Functions, Commun. Num. Theor. Phys. 11 (2017) 165 [arXiv:1512.06779] [INSPIRE].

[16] E. D'Hoker and D.H. Phong, Two loop superstrings. 1. Main formulas, Phys. Lett. B 529 (2002) 241 [hep-th/0110247] [INSPIRE].

[17] E. D'Hoker and D.H. Phong, Lectures on two loop superstrings, Conf. Proc. C 0208124 (2002) 85 [hep-th/0211111] [INSPIRE].

[18] E. D'Hoker and D.H. Phong, Two-loop superstrings VI: Non-renormalization theorems and the 4-point function, Nucl. Phys. B 715 (2005) 3 [hep-th/0501197] [INSPIRE]. 
[19] E. D'Hoker and M.B. Green, Zhang-Kawazumi Invariants and Superstring Amplitudes, arXiv: 1308.4597 [INSPIRE].

[20] E. D'Hoker, M.B. Green, B. Pioline and R. Russo, Matching the $D^{6} R^{4}$ interaction at two-loops, JHEP 01 (2015) 031 [arXiv:1405.6226] [INSPIRE].

[21] E. D'Hoker, M.B. Green and B. Pioline, Higher genus modular graph functions, string invariants and their exact asymptotics, to appear in Commun. Math. Phys. (2018) [arXiv:1712.06135] [INSPIRE].

$[22]$ B. Pioline and R. Russo, Infrared divergences and harmonic anomalies in the two-loop superstring effective action, JHEP 12 (2015) 102 [arXiv:1510.02409] [INSPIRE].

[23] E. D'Hoker, M.B. Green and B. Pioline, Asymptotics of the $D^{8} \mathcal{R}^{4}$ genus-two string invariant, Commun. Num. Theor. Phys. 13 (2019) [arXiv:1806.02691] [inSPIRE].

[24] M.B. Green, J.G. Russo and P. Vanhove, Modular properties of two-loop maximal supergravity and connections with string theory, JHEP 07 (2008) 126 [arXiv:0807.0389] [INSPIRE].

[25] Z. Bern, L.J. Dixon, D.C. Dunbar, M. Perelstein and J.S. Rozowsky, On the relationship between Yang-Mills theory and gravity and its implication for ultraviolet divergences, Nucl. Phys. B 530 (1998) 401 [hep-th/9802162] [INSPIRE].

[26] Z. Bern, J.J.M. Carrasco, L.J. Dixon, H. Johansson and R. Roiban, Manifest Ultraviolet Behavior for the Three-Loop Four-Point Amplitude of $N=8$ Supergravity, Phys. Rev. D 78 (2008) 105019 [arXiv:0808.4112] [INSPIRE].

[27] D. Zagier, The Rankin-Selberg method for automorphic functions which are not of rapid decay, J. Fac. Sci. Univ. Tokyo Sect. IA Math. 28 (1981) 415.

[28] A. Basu, Non-BPS interactions from the type-II one loop four graviton amplitude, Class. Quant. Grav. 33 (2016) 125028 [arXiv:1601.04260] [INSPIRE].

[29] K. Bringmann, N. Diamantis and S. Ehlen, Regularized inner products and errors of modularity, Int. Math. Res. Not. 2017 (2016) 7420.

[30] B. Pioline, A Theta lift representation for the Kawazumi-Zhang and Faltings invariants of genus-two Riemann surfaces, J. Number Theor. 163 (2016) 520 [arXiv:1504.04182] [INSPIRE].

[31] N. Kawazumi, Johnson's homomorphisms and the Arakelov-Green function, arXiv:0801.4218.

[32] S.-W. Zhang, Gross-Schoen cycles and dualising sheaves, Invent. Math. 179 (2010) 1.

[33] E. Gottschling, Explizite Bestimmung der Randflächen des Fundamentalbereiches der Modulgruppe zweiten Grades, Math. Ann. 138 (1959) 103.

[34] I. Florakis and B. Pioline, On the Rankin-Selberg method for higher genus string amplitudes, Commun. Num. Theor. Phys. 11 (2017) 337 [arXiv: 1602.00308] [InSPIRE].

[35] A. Basu, Perturbative type-II amplitudes for BPS interactions, Class. Quant. Grav. 33 (2016) 045002 [arXiv: 1510.01667] [INSPIRE].

[36] N. Berkovits, Super-Poincaré covariant two-loop superstring amplitudes, JHEP 01 (2006) 005 [hep-th/0503197] [INSPIRE].

[37] H. Gomez and C.R. Mafra, The closed-string 3-loop amplitude and S-duality, JHEP 10 (2013) 217 [arXiv: 1308.6567] [INSPIRE]. 
[38] G. Bossard and A. Kleinschmidt, Supergravity divergences, supersymmetry and automorphic forms, JHEP 08 (2015) 102 [arXiv:1506.00657] [INSPIRE].

[39] S. Stieberger, Constraints on Tree-Level Higher Order Gravitational Couplings in Superstring Theory, Phys. Rev. Lett. 106 (2011) 111601 [arXiv:0910.0180] [InSPIRE].

[40] O. Schlotterer and S. Stieberger, Motivic Multiple Zeta Values and Superstring Amplitudes, J. Phys. A 46 (2013) 475401 [arXiv:1205.1516] [inSPIRE].

[41] M.B. Green, C.R. Mafra and O. Schlotterer, Multiparticle one-loop amplitudes and S-duality in closed superstring theory, JHEP 10 (2013) 188 [arXiv:1307.3534] [INSPIRE].

[42] P. Tourkine, Tropical Amplitudes, Annales Henri Poincaré 18 (2017) 2199 [arXiv: 1309.3551] [INSPIRE].

[43] K. Bringmann, B. Kane and M. Viazovska, Theta lifts and local Maass forms, Math. Res. Lett. 20 (2013) 213.

[44] K. Bringmann and B. Kane, Modular local polynomials, Math. Res. Lett. 23 (2016) 973.

[45] D. Zagier, unpublished notes (2008).

[46] S. Wolpert, Asymptotics of the Spectrum and the Selberg Zeta Function on the Space of Riemann Surfaces, Commun. Math. Phys. 112 (1987) 283.

[47] S.F. Moosavian and R. Pius, Hyperbolic Geometry and Closed Bosonic String Field Theory I: The String Vertices Via Hyperbolic Riemann Surfaces, arXiv:1706.07366 [INSPIRE].

[48] J. Fay, Theta functions on Riemann surfaces, Lectures Notes in Mathematics, vol. 352, Springer, Berlin, Germany (1973).

[49] B. Zwiebach, How covariant closed string theory solves a minimal area problem, Commun. Math. Phys. 136 (1991) 83 [INSPIRE]. 